\title{
Multi-layer flows of immiscible fractional second grade fluids in a rectangular channel
}

\author{
Abdul Rauf ${ }^{1}\left[\right.$. Aoun Muhammad ${ }^{1}$ \\ Received: 2 May 2020 / Accepted: 3 September 2020 / Published online: 21 September 2020 \\ (c) Springer Nature Switzerland AG 2020
}

\begin{abstract}
Multi-layer laminar unsteady flows of immiscible fractional second grade fluids in a rectangular channel made by two parallel plates are studied. The fluid motion is produced by the motion of parallel walls in their plane and by the timedependent pressure gradient in the presence of the linear fluid-fluid interface conditions. The mathematical model is based on the generalized constitutive equations for the shear stress described by the time-fractional Caputo derivative. Integral transforms (finite Fourier sine transform and Laplace transform) have been used to obtain analytical and semi-analytical solutions for velocity, shear stress and the temperature fields. In the case of semi-analytical solutions, the Talbot's algorithms are used for the inverse Laplace transform. The numerical calculations are carried out with the help of Mathcad software, and the results are illustrated graphically. It has been found that the memory effects have a significant influence on the motion of the fluids.
\end{abstract}

Keywords n-layered immiscible fluids . Fractional second grade fluids . Analytical and semi analytical solutions . Integral transforms

Mathematics Subject Classification 76-XX · 76T30 -76D50

\section{Nomenclature}

$\rho_{i}$

$\mu_{i}$

$v_{i}$

$u_{0}$

$G_{i}$

$\tau_{i}$

$u_{i}(y, t)$

$P$

$h$

$\bar{X}(y, \xi)$

$E_{\alpha_{i} \beta_{i}}(\cdot)$

$G_{\sigma_{1}, \sigma_{2}, \sigma_{3}}(t, \sigma)$
Density

Dynamic viscosity

Kynamic viscosity

Characteristic velocity

Elastic modulus

Shear stress

Velocity

Pressure

Distance between two plates

Laplace transform of the function $X(y, t)$

Mittag-Leffler function

G-Lorenzo Hartely function

\section{Introduction}

Flows of immiscible materials in the channel/pipe are commonly found in nature. The study of simultaneous flow of two or more immiscible fluids is significant due to its wide applications in science, medical, geophysics, industry, petroleum engineering and hydrogeology [1-4]. Various applications include oil recovery, blood flow through capillary vessels, equipment cleaning, bio-films and mucus flow in living cells, removal of carbon dioxide from the atmosphere, groundwater management, crude oil flow through pipelines, bubble generation in microfluidics and bubble trains flow in various complex porous systems.

Several researchers have studied the stability/instability of two-layer or multi-layer immiscible fluids flow [5-7]. The linear stability of the viscoelastic two-layered plane

Abdul Rauf, attari_ab092@yahoo.com; abdul.rauf@aumc.edu.pk | Department of Computer Science and Engineering, Air University Multan Campus, Multan, Pakistan. 
Poiseuille and Couette flows have been first studied by Yih [8] with the help of long-wave approach. He observed that both density and viscosity stratification can cause interfacial Kelvin-Helmholtz instability. Herve Le Meur [9] has studied the uniqueness and the existence of the multilayered Poiseuille/ Couette fluid flow in pipes/channel and observed that interpolated Oldroyd derivative parameter and the viscosity ratios are significant for a unique solution. Kalogirou and Blyth [10] have considered the Couette-Poiseuille flow of the two-layered superposed fluids to discuss stability. The fluid at the lower layer is populated with surfactants and these surfactants get adsorbed on the interface. It has been observed that if the thickness ratio is much higher than the fluid viscosity ratio and if the surfactant is sufficiently soluble, the flow is stable.

In [11], Kim et al. have worked on the two-layered immiscible Couette flow with the help of a hybrid method. The flow is between two parallel planes in which the upper plane is moving while the lower plane is kept stationary. It has been found that the viscosity ratio has strong effects on the fluid velocity than the surface energy. Two layer simultaneous natural convectional flow in a vertical semi-corrugated channel under the magnetic effects have been investigated by Abd Elmaboud [12]. The flow domain consists of two subdomains, the first one contains the magneto nanofluids, and the other contains a pure non-conducting viscous fluid. The results demonstrate that in the absence of heat soures nad with the rise in the nano-particle volume fraction, theremal transfer enhancement has been observed in both sub-domains. Later, Abd Elmaboud et al. [13], have studied the electro-magnetic simultaneous two layers immiscible fluids flow over an inclined plate. The flow domain comprises two parts, one of which is made of porous matrix saturated in Newtonian fluid, and the other has clear fluid. The findings demonstrate that the electrical field raises the velocity profile of both subdomains. Whereas the velocity decreases by rising the magnetic field owing to the Lorentz power. In [14], Khan et al have investigated the heat transfer and the fluid velocity of the two-layer immiscible fluid in the presence of pressure type die. The first layer is filled with the inelastic fluid, namely, power-law fluid and the second layer is filled with the viscoelastic liquid (Phan-Thien-Tanner fluids). It has been seen that the fluid velocity and the fluid temperature increase with the increase in the Deborah number.

Hisham et al. [15], for example, presented an analytical study of the two-layer flow of immiscible Maxwell fluids between two parallel moving plates in the presence of time-dependent pressure gradient. Analytical solutions for velocities and shear stresses are recovered with the help of integral transforms, Laplace and finite Fourier sine transform. It has been found that the increase in kinematic viscosity decreases the maximum value of the velocity. Later Rauf et al. [16], found the analytical and the semianalytical solutions for the velocity fields and the temperature fields for the simultaneous flow of $n$-immiscible fractional Maxwell fluid in a rectangular channel bounded by the two parallel translating planes and in the presence of time-dependent pressure gradient. It has been observed that the thermal transport in the ordinary fluids is higher as compared with the fluids with the thermal memory, whereas the fractional parameters for the velocity fields act as accelerating factors of the fluids. Recently Rauf et al. [17], studied the simultaneous flow of n-immiscible fractional Maxwell fluid in a cylindrical domain. The motion is caused by the translational motion of the cylinder and in the presence of the time-dependent pressure gradient in the direction of the flow. Analytical solutions for the velocities and the shear stresses are obtained with the help of the Laplace transform coupled with the finite Weber transform of order zero and the finite Hankel transform of order zero. It has been seen that the fluid velocity decreases with the increase in the values of the fluids fractional parameters. Other interesting results relating to sigle layer fluid flow [18-21] and simultaneous flow two or more fluids can be seen in $[7,15,22-26]$. To the best of authors knowledge, the study of simultaneous flow of multi-layer immiscible second grade not exist in literature. In this paper, we have carried out this study.

Modeling of complex systems with the fractional order differential and integral operators have applications in many fields of science such as geophysics, biology, demography, bioengineering, physics and mathematics; see [27] and the references therein. There exist many fractional differential operators in literature such as CaputoFabrizio fractional derivative [27], Riemann-Liouville fractional integral/derivative [28], Caputo fractional derivative [29], and Yang-Srivastava-Machado fractional derivative [30], are some of the examples of the fractional-order differential operators used in viscoelasticity, mass and heat transport processes. Hristov [31] studied the transient space-fractional diffusion with power-law superdiffusivity modeled by the Riemann-Liouville fractional derivative. Ahmad et al. [32] have applied the time-fractional Caputo-Fabrizio derivative to study the two-dimensional advective diffusion process with concentrated source and the short-range memory.

In this paper, we have studied the multi-layer flow of immiscible fractional second grade fluids between two parallel plates. We have considered an unsteady, incompressible, and one dimensional fully developed flow which is generated by the movement of the boundary walls and the time-dependent pressure gradient in the presence of the generalized thermal flux within the fluid layers. Moreover, we have considered the linear interfacial fluid-fluid 
condition between two consecutive layers. To find analytical solutions for velocities and shear stresses we have used finite Fourier sine transform in conjunction with the Laplace transformation. The main advantage of the analytic solution is that comparing an analytical solution with the numerical scheme is the best way to examine the accuracy. A semi-analytical solution for temperature fields is recovered with the help of Laplace transform and Tablot's algorithms used for the numerical Laplace inversion.

\section{Mathematical modeling}

The flow domain for the simultaneous n-layer flow of the fractional second grade fluids is $D^{\prime}=\left\{\left(x^{\prime}, y^{\prime}, z^{\prime}\right),-\infty<x^{\prime}, z^{\prime}<\infty, 0 \leq y^{\prime} \leq h\right\}$ with the boundary walls situated in planes $y^{\prime}=0$ and $y^{\prime}=h>0$. Initially at time $t^{\prime}=0$ both boundary plates and the fluids enclosed inside them are at rest. After this moment, the boundary plate at $y^{\prime}=0$ start translating towards the $x^{\prime}$-axis with the velocity $u_{10}^{\prime}=U_{0} f_{1}\left(t^{\prime}\right)$, whereas the other plate at $y^{\prime}=h$ moves parallel to the $x^{\prime}$-axis with the velocity $u_{20}^{\prime}=U_{0} g_{1}\left(t^{\prime}\right)$ (Fig. 1 ), where $U_{0}$ is the characteristic velocity. We assumes that the functions $f_{1}\left(t^{\prime}\right) g_{1}\left(t^{\prime}\right)$ are piece-wise continuous functions with $f_{1}(0)=g_{1}(0)=0$. We assume that the n-layer flow of fluids is immiscible, fully developed, unsteady and one dimensional. The motion of the fluids is caused by the motion of the boundary walls and the time-dependent pressure gradient in the direction of the flow. Under the given conditions, fluids velocities take the form $\mathbf{v}_{i}^{\prime}=\left(u_{i}^{\prime}\left(y^{\prime}, t^{\prime}\right), 0,0\right)$. Let $h_{0}=0$ and $h_{n}=h$.
In the region $y^{\prime} \in\left[h_{i-1}, h_{i}\right], h_{i-1}<h_{i}$ flows a second grade fluid with viscosity $\mu_{i}$, the density $\rho_{i}, G_{i}$, the elastic modulus, velocity $u_{i}^{\prime}\left(y^{\prime}, t^{\prime}\right)$ and the share stress $\tau_{i}^{\prime}\left(y^{\prime}, t^{\prime}\right)$ where $i=1,2, \ldots, n$. We fix here the notations $I_{n}^{1}:=\{1,2, \ldots, n\}$, $I_{n-1}^{0}:=\{0,1, \ldots, n-1\}$. The continuity equation is identically satisfied by all velocities $u_{i}\left(y^{\prime}, t^{\prime}\right), i \in l_{n}^{1}$. The governing equations of motion along with initial, boundary and the fluid-fluid interfacial conditions are

- the system of linear momentum equations

$$
\rho_{i} \frac{\partial u_{i}^{\prime}}{\partial t^{\prime}}=\frac{\partial \tau_{i}^{\prime}}{\partial y^{\prime}}-\frac{\partial p^{\prime}}{\partial x^{\prime}}, \quad i \in I_{n^{\prime}}^{1}
$$

- the system of constitutive equations

$$
\tau_{i}^{\prime}=\left(\mu_{i}+\alpha_{i}^{\prime} \frac{\partial u_{i}^{\prime}}{\partial t^{\prime}}\right) \frac{\partial u_{i}^{\prime}}{\partial y^{\prime}}, i \in I_{n^{\prime}}^{1}
$$

- the initial conditions

$$
u_{i}^{\prime}\left(y^{\prime}, 0\right)=0, \tau_{i}^{\prime}\left(y^{\prime}, 0\right)=0, i \in I_{n}^{1}
$$

- the boundary conditions

$$
\begin{array}{ll}
\text { at } y^{\prime}=0, & u_{1}^{\prime}\left(0, t^{\prime}\right)=U_{0} f_{1}\left(t^{\prime}\right), \\
\text { at } y^{\prime}=h_{n}=h, & u_{n}^{\prime}\left(h, t^{\prime}\right)=U_{0} g_{1}\left(t^{\prime}\right),
\end{array}
$$

- the fluid-fluid interface conditions

$$
u_{i}^{\prime}\left(h_{i}, t^{\prime}\right)=u_{i+1}^{\prime}\left(h_{i}, t^{\prime}\right), \tau_{i}^{\prime}\left(h_{i}, t^{\prime}\right)=\tau_{i+1}^{\prime}\left(h_{i}, t^{\prime}\right), i \in l_{n-1}^{1}
$$

Fig. 1 Geometry of the problem

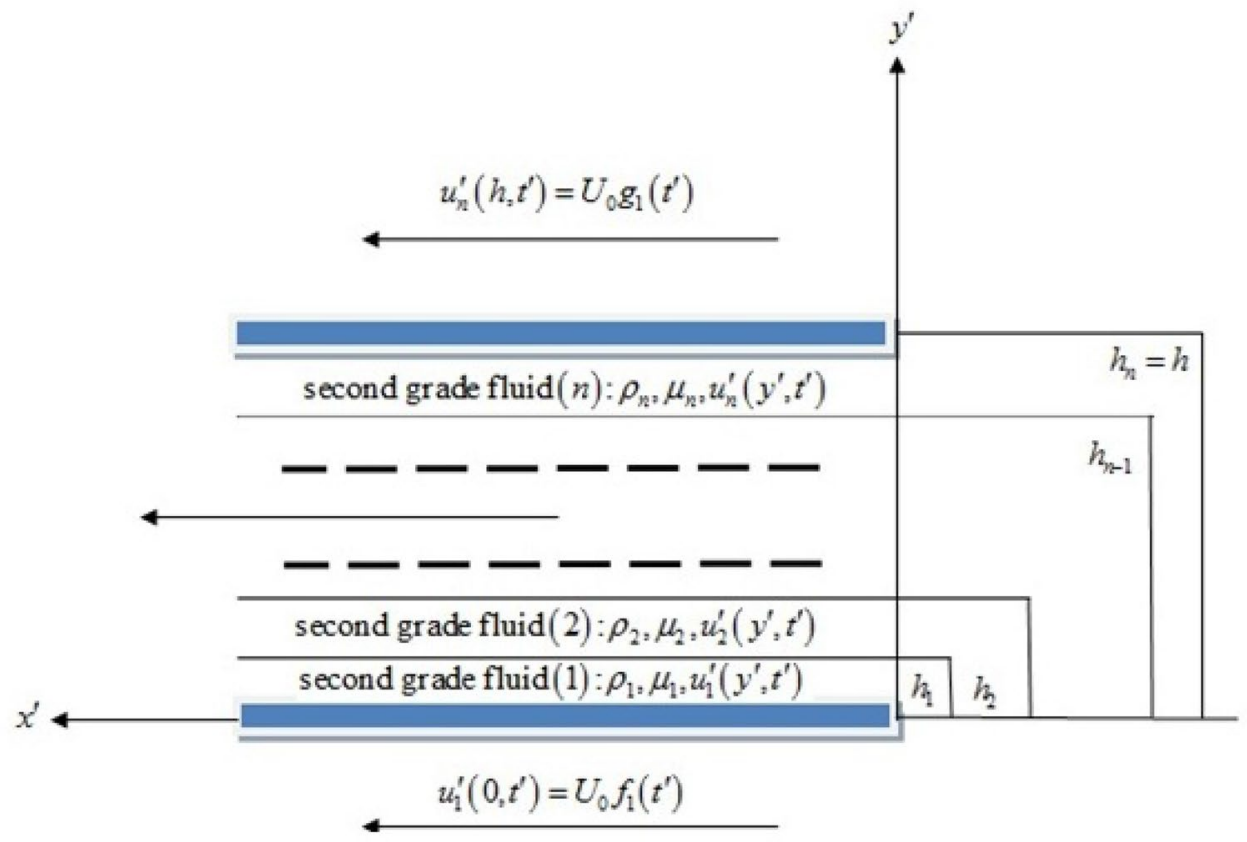


Consider the following non-dimensional parameters into Eqs. (1)-(5),

$$
\begin{aligned}
x & =\frac{x^{\prime}}{h}, y=\frac{y^{\prime}}{h}, t=\frac{v_{1} t^{\prime}}{h^{2}}, u_{i}=\frac{u_{i}^{\prime}}{U_{0}}, \tau_{i}=\frac{h \tau_{i}^{\prime}}{\mu_{1} U_{0}}, \\
v_{i} & =\frac{\mu_{i}}{\rho_{i}}, p=\frac{h p^{\prime}}{\mu_{1} U_{0}}, \\
\alpha_{i} & =\frac{\alpha^{\prime}{ }_{i} U_{0}}{\rho_{1} h^{2}}, a_{i}=\frac{\rho_{i}}{\rho_{1}}, b_{i}=\frac{\mu_{i}}{\mu_{1}}, \\
f(t) & =f_{1}\left(\frac{h^{2} t}{v_{1}}\right), g(t)=g_{1}\left(\frac{h^{2} t}{v_{1}}\right), \\
d_{i} & =\frac{h_{i}}{h}, \sigma_{i}=\frac{v_{i}}{v_{1}}, i \in I_{n^{\prime}}^{1}
\end{aligned}
$$

we get the following non-dimensional mathematical model for governing equations,

$a_{i} \frac{\partial u_{i}}{\partial t}=-\frac{\partial p}{\partial x}+\frac{\partial \tau_{i}}{\partial y}, i \in I_{n^{\prime}}^{1}$

$\tau_{i}=\left(b_{i}+\alpha_{i} \frac{\partial u_{i}}{\partial t}\right) \frac{\partial u_{i}}{\partial y}, i \in l_{n^{\prime}}^{1}$

along with the dimensionless initial conditions, the boundary conditions and the interface conditions

$u_{i}(y, 0)=0, \tau_{i}(y, 0)=0, i \in l_{n^{\prime}}^{1}$

$u_{1}(0, t)=f(t), u_{n}(1, t)=g(t)$,

the interface conditions

$u_{i}\left(d_{i}, t\right)=u_{i+1}\left(d_{i}, t\right), \tau_{i}\left(d_{i}, t\right)=\tau_{i+1}\left(d_{i}, t\right), i \in I_{n-1}^{1}$.

\subsection{Fractional mathematical model for constitutive equations}

Consider the following generalized constitutive mathematical relation,

$\tau_{i}=b_{i} \frac{\partial u_{i}}{\partial y}+\alpha_{i} \mathfrak{D}_{t}^{\gamma_{i}} \frac{\partial u_{i}}{\partial y}, \gamma_{i} \in(0,1], i \in l_{n^{\prime}}^{1}$

where the Caputo derivative $\mathfrak{D}_{t}^{\sigma}$ is defined as, [17],

$\mathfrak{D}_{t}^{\sigma} \mathfrak{F}(y, t)=\frac{1}{\Gamma(1-\sigma)} \int_{0}^{t}(t-\varrho)^{-\sigma} \frac{\partial \mathfrak{F}(y, \varrho)}{\partial \varrho} d \varrho, 0 \leq \sigma<1$
For the special case $\sigma=1, \mathfrak{D}_{t}^{1} \mathfrak{F}(y, t)=\frac{\partial \mathfrak{F}(y, t)}{\partial t}$. Let $(\mathfrak{S}(y, \xi)$ be the Laplace transform of the $\mathfrak{F}(y, t)$ with $\mathfrak{F}(y, 0)=0$, then the Laplace transform of the Caputo derivative is defined by $L\left\{\mathfrak{D}_{t}^{\sigma} \mathfrak{F}(y, t)\right\}=\xi^{\sigma}(\mathfrak{S}(y, \xi)$, where $0 \leq \sigma<1$ [33]. The Laplace transformation of the Caputo derivative It is significant to remark that the generalized model (11) have the following equivalent formulae as

$$
\begin{aligned}
& \frac{\partial u_{i}(y, t)}{\partial y}=\psi_{i}\left(t, \gamma_{i}\right) * \tau_{i}(y, t) \\
& =\int_{0}^{t} \psi_{i}\left(t-\delta, \gamma_{i}\right) \tau_{i}(y, \delta) d \delta, \quad i \in I_{n^{\prime}}^{1}
\end{aligned}
$$

where $\psi_{i}\left(t, \gamma_{i}\right)=\frac{1}{\alpha_{i}} t^{\gamma_{i}-1} E_{\gamma_{i}, \gamma_{i}}\left(-\frac{b_{i}}{\alpha_{i}} t_{i}\right), i \in I_{n}^{1}$, is the velocity gradient non-locality kernel. Here in the flow direction the pressure gradient is the known function

$-\frac{\partial p}{\partial x}=P(t)$

where $P(t)$ is the piece-wise continuous function over positive real line.

\section{Solution of the problem}

We have used the Laplace transform coupled with the finite sine-Fourier transform [34] for the analytical solutions of the Eqs. (6), (11) with conditions (8)-(10). With the application of the Laplace transform to Eqs. (6), (9)-(11) in the presence of the initial conditions (8), we obtain

$a_{i} \xi \bar{u}_{i}(y, \xi)=\bar{P}(\xi)+\frac{\partial \bar{\tau}_{i}(y, \xi)}{\partial y}, i \in I_{n^{\prime}}^{1}$

$\bar{\tau}_{i}(y, \xi)=\left(b_{i}+\alpha_{i} \xi^{\gamma_{i}}\right) \frac{\partial \bar{u}_{i}(y, \xi)}{\partial y}, i \in l_{n^{\prime}}^{1}$

$\bar{u}_{1}(0, \xi)=\bar{f}(\xi), \bar{u}_{n}(1, \xi)=\bar{g}(\xi)$

$\bar{u}_{j}\left(d_{j}, \xi\right)=\bar{u}_{j+1}\left(d_{j}, \xi\right), \bar{\tau}_{j}\left(d_{j}, \xi\right)$

where $\bar{\chi}(y, \xi)=\int_{0}^{\infty} \chi(y, t) \exp (-\xi t) d t$ is the Laplace transform of the function $\chi(y, t)$ [34]. 


\subsection{Analytical solutions for velocities and shear stresses}

By using Eqs. (16) and (17) we get the Laplace transformed n-layer velocities,

$a_{i} \xi \bar{u}_{i}(y, \xi)=\bar{P}(\xi)+\left(b_{i}+\alpha_{i} \xi^{\gamma_{i}}\right) \frac{\partial^{2} \bar{u}_{i}(y, \xi)}{\partial y^{2}}, i \in l_{n^{\prime}}^{1}$

We will further apply the finite sine-Fourier transform to Eq. (20) for which the function $\bar{\chi}(y, \xi), y \in[a, b], a<b$, is defined as [35]

$$
\begin{aligned}
\widetilde{\bar{\chi}}_{m}(\xi) & =\int_{a}^{b} \bar{\chi}(y, \xi) \sin \left(\vartheta_{m}(y-a)\right) d y, \\
\vartheta_{m} & =\frac{m \pi}{b-a}, m=1,2,3, \ldots,
\end{aligned}
$$

along with the inverse Fourier transform defined by

$\bar{\chi}(y, \xi)=\frac{2}{b-a} \cdot \sum_{m=1}^{\infty} \widetilde{\bar{\chi}}_{m}(\xi) \sin \left(\vartheta_{m}(y-a)\right)$.

With the application of finite Fourier sine transformation (21) to Eq. (20) along with the boundary conditions (18) and interface fluid-fluid condition (19), the transformed velocities take the form

$$
\begin{aligned}
& \tilde{\tilde{u}}_{i m}(\xi)= \frac{\left[1-(-1)^{m}\right]}{\vartheta_{m}^{(i)}} \frac{\bar{P}(\xi)}{a_{i} \xi+\left(b_{i}+\alpha_{i} \xi \gamma_{i}\right)\left(\vartheta_{m}^{(i)}\right)^{2}} \\
&+\frac{\vartheta_{m}^{(i)}\left(b_{i}+\alpha_{i} \xi^{\gamma_{i}}\right)\left(\bar{u}_{i}\left(d_{i-1}, \xi\right)\right)}{a_{i} \xi+\left(b_{i}+\alpha_{i} \xi\right)\left(\vartheta_{m}^{(i)}\right)^{2}} \\
&-\frac{\vartheta_{m}^{(i)}\left(b_{i}+\alpha_{i} \xi^{\gamma_{i}}\right)(-1)^{m} \bar{u}_{i}\left(d_{i}, \xi\right)}{a_{i} \xi+\left(b_{i}+\alpha_{i} \xi \gamma_{i}\right)\left(\vartheta_{m}^{(i)}\right)^{2}} \\
& \vartheta_{m}^{(i)}=\frac{m \pi}{d_{i}-d_{i-1}}, m=1,2, \ldots, i \in l_{n^{\prime}}^{1}
\end{aligned}
$$

where for $i=1, \bar{u}_{1}\left(d_{0}, \xi\right)=\bar{f}(\xi)$ and for $i=n, \bar{u}_{n}\left(d_{n}, \xi\right)=\bar{g}(\xi)$. In order to apply the inverse Fourier sine transform, we rewrite the Eq. (23) in the following suitable form

$$
\begin{aligned}
& \tilde{\tilde{u}}_{i m}(\xi)= \frac{(-1)^{m+1} \bar{u}_{i}\left(d_{i}, \xi\right)}{\vartheta_{m}^{(i)}}+\frac{\bar{u}_{i}\left(d_{i-1}, \xi\right)}{\vartheta_{m}^{(i)}} \\
&+\frac{\left[1-(-1)^{m}\right]}{\vartheta_{m}^{(i)}} \frac{\bar{P}(\xi)}{a_{i} \xi+\left(b_{i}+\alpha_{i} \xi^{\gamma_{i}}\right)\left(\vartheta_{m}^{i}\right)^{2}} \\
&-\frac{a_{i} \xi \bar{u}_{i}\left(d_{i-1}, \xi\right)}{\vartheta_{m}^{i}\left[a_{i} \xi+\left(b_{i}+\alpha_{i} \xi\right)\left(\vartheta_{m}^{(i)}\right)^{2}\right]} \\
&+\frac{a_{i} \xi(-1)^{m} \bar{u}_{i}\left(d_{i}, \xi\right)}{\vartheta_{m}^{(i)}\left[a_{i} \xi+\left(b_{i}+\alpha_{i} \xi^{\gamma_{i}}\right)\left(\vartheta_{m}^{(i)}\right)^{2}\right]} \\
& \vartheta_{m}^{(i)}=\frac{m \pi}{d_{i}-d_{i-1}}, m=1,2, \ldots, i \in l_{n}^{1} .
\end{aligned}
$$

Consider the auxiliary functions $\wp_{1 i}(y), \wp_{2 i}(y)$ and their inverse Fourier sine transforms $\tilde{\mathscr{\wp}}_{1 \text { im }}, \tilde{\mathscr{\delta}}_{2 i m}$

$\wp_{1 i}(y)=\frac{d_{i}-y}{d_{i}-d_{i-1}}, y \in\left[d_{i-1}, d_{i}\right], \tilde{\wp}_{1 i m}=\frac{1}{\vartheta_{m}^{(i)}}$

$\wp_{2 i}(y)=\frac{y-d_{i-1}}{d_{i}-d_{i-1}}, y \in\left[d_{i-1}, d_{i}\right], \tilde{\wp}_{2 i m}=\frac{(-1)^{m+1}}{\vartheta_{m}^{(i)}}$

$m=1,2, \ldots, i \in l_{n}^{1}, d_{0}=0, d_{n}=1$.

The inverse Fourier sine transform of Eq. (24) takes the form

$$
\begin{aligned}
\bar{u}_{i}(y, \xi)= & \wp_{1 i}(y) \bar{u}_{i}\left(d_{i-1}, \xi\right)+\wp_{2 i}(y) \bar{u}_{i}\left(d_{i}, \xi\right) \\
& +\frac{2}{d_{i}-d_{i-1}} \sum_{m=1}^{\infty} \sin \left(\vartheta_{m}^{(i)}\left(y-d_{i-1}\right)\right) \\
& \frac{\left[1-(-1)^{m}\right]}{\vartheta_{m}^{(i)}} \frac{\bar{P}(\xi)}{a_{i} \xi+\left(b_{i}+\alpha_{i} \xi_{i}\right)\left(\vartheta_{m}^{i}\right)^{2}} \\
& -\frac{2}{d_{i}-d_{i-1}} \sum_{m=1}^{\infty} \sin \left(\vartheta_{m}^{(i)}\left(y-d_{i-1}\right)\right) \\
& \frac{a_{i} \xi \bar{u}_{i}\left(d_{i-1}, \xi\right)}{\vartheta_{m}^{i}\left[a_{i} \xi+\left(b_{i}+\alpha_{i} \xi \gamma_{i}\right)\left(\vartheta_{m}^{(i)}\right)^{2}\right]} \\
& +\frac{2}{d_{i}-d_{i-1}} \sum_{m=1}^{\infty} \sin \left(\vartheta_{m}^{(i)}\left(y-d_{i-1}\right)\right) \\
& \frac{a_{i} \xi(-1)^{m} \bar{u}_{i}\left(d_{i}, \xi\right)}{\vartheta_{m}^{(i)}\left[a_{i} \xi+\left(b_{i}+\alpha_{i} \xi_{i}\right)\left(\vartheta_{m}^{(i)}\right)^{2}\right]}
\end{aligned}
$$


$\vartheta_{m}^{(i)}=\frac{m \pi}{d_{i}-d_{i-1}}, m=1,2, \ldots, i \in l_{n}^{1}$.

Now, from (17) and (26), we obtain

$$
\begin{aligned}
\bar{\tau}_{i}(y, \xi)= & \left(b_{i}+\alpha_{i} \xi^{\gamma_{i}}\right) \frac{\partial \bar{u}_{i}(y, \xi)}{\partial y}=\bar{T}_{i 1}(y, \xi) \bar{u}_{i}\left(d_{i}, \xi\right) \\
& -\bar{T}_{i 2}(y, \xi) \bar{u}_{i}\left(d_{i-1}, \xi\right)+\bar{T}_{i 3}(y, \xi) \bar{P}(\xi),
\end{aligned}
$$

where

$$
\begin{aligned}
\bar{T}_{i 1}(y, \xi)= & \frac{\left(b_{i}+\alpha_{i} \xi^{\gamma_{i}}\right)}{d_{i}-d_{i-1}} \\
& \left(1+2 \sum_{m=1}^{\infty} \frac{a_{i}(-1)^{m} \xi \cos \left(\vartheta_{m}^{(i)}\left(y-d_{i-1}\right)\right)}{\left[a_{i} \xi+\left(b_{i}+\alpha_{i} \xi^{\gamma_{i}}\right)\left(\vartheta_{m}^{i}\right)^{2}\right]}\right), \\
\bar{T}_{i 2}(y, \xi)= & \frac{\left(b_{i}+\alpha_{i} \xi^{\gamma_{i}}\right)}{d_{i}-d_{i-1}} \\
& \left(\begin{array}{l}
\left.1+2 \sum_{m=1}^{\infty} \frac{a_{i} \xi \cos \left(\vartheta_{m}^{(i)}\left(y-d_{i-1}\right)\right)}{\left[a_{i} \xi+\left(b_{i}+\alpha_{i} \xi \gamma_{i}\right)\left(\vartheta_{m}^{i}\right)^{2}\right]}\right), \\
\bar{T}_{i 3}(y, \xi)= \\
\\
\left(\sum_{m=1}^{\infty} \frac{\left[1-\left(b_{i}+\alpha_{i} \xi^{\gamma_{i}}\right)\right.}{d_{i}-d_{i-1}}\right. \\
{\left[a_{i} \xi+\left(b_{i}+\alpha_{i} \xi^{m}\right)\left(\vartheta_{m}^{i}\right)^{2}\right]}
\end{array}\right), i \in l_{n}^{1} .
\end{aligned}
$$

With the help of the interface liquid-liquid conditions (19) in Eqs. (27) and (28), we obtained the following algebraic system for the Laplace transformed velocities on the interfaces $y=d_{i}, i=1,2, \ldots, n-1$,

$$
\begin{aligned}
& -\overline{\mathbf{e}}_{1,1}(\xi) \bar{u}_{1}\left(d_{1}, \xi\right)+\overline{\mathbf{e}}_{1,2}(\xi) \bar{u}_{2}\left(d_{2}, \xi\right)=\bar{b}_{1}(\xi), \\
& \overline{\mathbf{e}}_{i, i-1}(\xi) \bar{u}_{i-1}\left(d_{i-1}, \xi\right)-\overline{\mathbf{e}}_{i, i}(\xi) \bar{u}_{i}\left(d_{i}, \xi\right)+\overline{\mathbf{e}}_{i, i+1}(\xi) \bar{u}_{i+1}\left(d_{i+1}, \xi\right) \\
& \quad=\bar{b}_{i}(\xi), \quad i \in J_{n-2^{\prime}}^{(2)} \\
& \overline{\mathbf{e}}_{n-1, n-2}(\xi) \bar{u}_{n-2}\left(d_{n-2}, \xi\right)-\overline{\mathbf{e}}_{n-1, n-1}(\xi) \bar{u}_{n-1}\left(d_{n-1}, \xi\right)=\bar{b}_{n-1}(\xi),
\end{aligned}
$$

where

$$
\begin{aligned}
\overline{\mathbf{e}}_{1,1}(\xi) & =\bar{T}_{1,1}\left(d_{1}, \xi\right)+\bar{T}_{1,2}\left(d_{1}, \xi\right), \overline{\mathbf{e}}_{1,2}=\bar{T}_{1,2}\left(d_{1}, \xi\right), \\
\overline{\mathbf{e}}_{i, i-1}(\xi) & =\bar{T}_{i, 2}\left(d_{i}, \xi\right), \overline{\mathbf{e}}_{i, i}=\bar{T}_{i, 1}\left(d_{i}, \xi\right)+\bar{T}_{i+1,2}\left(d_{i}, \xi\right), \\
\overline{\mathfrak{e}}_{i, i+1}(\xi) & =\bar{T}_{i+1,1}\left(d_{i}, \xi\right), \quad i \in J_{n-1^{\prime}}^{(2)}
\end{aligned}
$$

and

$\bar{b}_{j}(\xi)=\bar{T}_{j, 3}\left(d_{j}, \xi\right)-\bar{T}_{j+1,3}\left(d_{j}, \xi\right)-\delta_{j, n-1} \bar{g}(\xi)-\delta_{j, 1} \bar{f}(\xi), \quad j \in J_{n-1}^{(1)}$, $\delta_{j, m-1}$ is Kronecker delta. The system (29) can be written in the following equivalent form

$\bar{M}(\xi) \bar{U}(\xi)=\bar{N}(\xi)$,

where

$\bar{M}(\xi)=\left[\begin{array}{cccccc}-\bar{f}(\xi) & \overline{\mathrm{e}}_{1,2}(\xi) & 0 & 0 & \ldots & 0 \\ \overline{\mathrm{e}}_{2,1}(\xi) & -\overline{\mathrm{e}}_{2,2}(\xi) & \overline{\mathrm{e}}_{2,3}(\xi) & 0 & \ldots & 0 \\ 0 & \overline{\mathrm{e}}_{3,2}(\xi) & -\overline{\mathrm{e}}_{3,3}(\xi) & \overline{\mathrm{e}}_{3,4}(\xi) & \ldots & \\ \vdots & \vdots & \ddots & \ddots & \ddots & \vdots \\ 0 & 0 & \ldots & -\overline{\mathrm{e}}_{n-3, n-3}(\xi) & \overline{\mathrm{e}}_{n-3, n-2}(\xi) & 0 \\ 0 & 0 & \ldots & \overline{\mathrm{e}}_{n-2, n-3}(\xi) & -\overline{\mathrm{e}}_{n-2, n-2}(\xi) & \overline{\mathrm{e}}_{n-2, n-1}(\xi) \\ 0 & 0 & \ldots & 0 & \overline{\mathrm{e}}_{n-1, n-2}(\xi) & -\bar{g}(\xi)\end{array}\right]$,

$\bar{U}(\xi)=\left[\begin{array}{c}\bar{u}_{1}\left(d_{1}, \xi\right) \\ \bar{u}_{2}\left(d_{2}, \xi\right) \\ \vdots \\ \bar{u}_{n-1}\left(d_{n-1}, \xi\right)\end{array}\right]$,

and

$\bar{N}(\xi)=\left[\begin{array}{l}\bar{b}_{1}(\xi) \\ \bar{b}_{2}(\xi) \\ \vdots \\ \bar{b}_{n-1}(\xi)\end{array}\right]$.

Finally, we obtain

$\bar{U}(\xi)=\bar{M}^{-1}(\xi) \bar{N}(\xi)$.

Now, $\bar{u}_{i}\left(d_{i}, \xi\right), i \in J_{n}^{(1)}$ are known functions, therefore the velocities $\bar{u}_{1}(y, \xi), \cdots, \bar{u}_{n}(y, \xi)$ are known. To obtained the inverse Laplace transforms of the functions $\bar{u}_{i}(y, \xi), i \in I_{n}^{1}$ we consider the following auxiliary functions,

$$
\begin{aligned}
\bar{H}_{i 0}(m, \xi) & =\frac{1}{a_{i} \xi+\left(b_{i}+\alpha_{i} \xi^{\gamma_{i}}\right)\left(\vartheta_{m}^{(i)}\right)^{2}} \\
& =\frac{\xi^{-\gamma_{i}}}{\left(a_{i}\right)\left(\xi^{1-\gamma_{i}}+\frac{\alpha_{i}\left(\vartheta_{m}^{(i)}\right)^{2}}{a_{i}}\right)} \cdot \frac{1}{1+\frac{b_{i}\left(\vartheta_{m}^{(i)}\right)^{2} \xi^{-\gamma_{i}}}{a_{i}\left(\xi^{1-\gamma_{i}+\frac{\alpha_{i}\left(\vartheta_{m}^{(i)}\right.}{a_{i}}}\right)}} \\
& =\sum_{k=0}^{\infty}(-1)^{k} \frac{b_{i}^{k}\left(\vartheta_{m}^{(i)}\right)^{2 k}}{a_{i}^{k+1}} \frac{\xi^{-(k+1) \gamma_{i}}}{\left(\xi^{1-\gamma_{i}}+\frac{\alpha_{i}\left(\vartheta_{m}^{(i)}\right)^{2}}{a_{i}}\right)^{k+1}}
\end{aligned}
$$




$$
\begin{aligned}
\bar{H}_{i 3}(m, \xi)= & \xi \bar{H}_{i 0}(m, \xi) \\
= & \frac{\xi^{-\gamma_{i}+1}}{a_{i}\left(\xi^{1-\gamma_{i}}+\frac{\alpha_{i}\left(\theta_{m}^{i}\right)^{2}}{a_{i}}\right)} \\
& +\sum_{k=0}^{\infty} \frac{(-1)^{k+1}\left(b_{i}\right)^{k+1}\left(\theta_{m}^{(i)}\right)^{2 k+2}}{a_{i}^{k+2}} \\
& \cdot \frac{\xi^{-(k+2) \gamma_{i}+1}}{\left(\xi^{1-\gamma_{i}}+\frac{\alpha_{i}\left(\theta_{m}^{(i)}\right)^{2}}{a_{i}}\right)^{k+2}}
\end{aligned}
$$

$$
\begin{aligned}
\bar{H}_{i 1}(m, \xi)= & \left(b_{i}+\alpha_{i} \xi^{\gamma_{i}}\right) \bar{H}_{i 0}(m, \xi) \\
= & \sum_{k=0}^{\infty} \frac{(-1)^{k}\left(b_{i}\right)^{k+1}\left(\theta_{m}^{(i)}\right)^{2 k}}{a_{i}^{k+1}} \\
& \cdot \frac{\xi^{-(k+1) \gamma_{i}}}{\left(\xi^{1-\gamma_{i}}+\frac{\alpha_{i}\left(\theta_{m}^{(i)}\right)^{2}}{a_{i}}\right)^{k+1}} \\
& +\sum_{k=0}^{\infty} \frac{(-1)^{k}\left(b_{i}\right)^{k+1}\left(\theta_{m}^{(i)}\right)^{2 k}\left(\alpha_{i}\right)}{a_{i}^{k+1}} \\
& \cdot \frac{\xi^{-k \gamma_{i}}}{\left(\xi^{1-\gamma_{i}}+\frac{\alpha_{i}\left(\theta_{m}^{(i)}\right)^{2}}{a_{i}}\right)^{k+1}}
\end{aligned}
$$$$
\bar{H}_{i 2}(m, \xi):=\xi \bar{H}_{i 1}(m, \xi)
$$$$
=\frac{\xi^{-\gamma_{i}+1}}{a_{i}\left(\xi^{1-\gamma_{i}}+\frac{\alpha_{i}\left(\theta_{m}^{i}\right)^{2}}{a_{i}}\right)}
$$$$
+\sum_{k=0}^{\infty} \frac{(-1)^{k+1}\left(b_{i}\right)^{k+1}\left(\theta_{m}^{(i)}\right)^{2 k+2}}{a_{i}^{k+2}}
$$$$
\frac{\xi^{-(k+2) \gamma_{i}+1}}{\left(\xi^{1-\gamma_{i}}+\frac{\alpha_{i}\left(\theta_{m}^{(i)}\right)^{2}}{a_{i}}\right)^{k+2}}
$$$$
+\frac{\alpha_{i} \xi}{a_{i}\left(\xi^{1-\gamma_{i}}+\frac{\alpha_{i}\left(\theta_{m}^{i}\right)^{2}}{a_{i}}\right)}
$$$$
+\sum_{k=0}^{\infty} \frac{(-1)^{k+1}\left(b_{i}\right)^{k+2}\left(\theta_{m}^{(i)}\right)^{2 k+2}\left(\alpha_{i}\right)}{a_{i}^{k+2}}
$$$$
\cdot \frac{\xi^{-\left((k+1) \gamma_{i}-1\right)}}{\left(\xi^{1-\gamma_{i}}+\frac{\alpha_{i}\left(\theta_{m}^{(i)}\right)^{2}}{a_{i}}\right)^{k+2}} \text {. }
$$

and

$\operatorname{Re}(s)>0, \operatorname{Re}\left(\sigma_{1} \sigma_{3}-\sigma_{2}\right)>0,\left|\frac{\sigma}{s^{\sigma_{1}}}\right|<1$

and for $\alpha_{i}, \beta_{i}>0$,

$\mathcal{L}^{-1}\left[\frac{s^{\alpha_{i}-\beta_{i}}}{s^{\alpha_{i}}-d}\right]=t^{\beta_{i}-1} E_{\alpha_{i}, \beta_{i}}\left(d t^{\alpha_{i}}\right)$,

where $E_{\alpha_{i}, \beta_{i}}($.$) is the Mittag-Leffler function and G_{\sigma_{1}, \sigma_{2}, \sigma_{3}}(t, \sigma)$ is the generalized G-Lorenzo-Hartley function [36]. The inverse Laplace transform of $\bar{H}_{i 0}(m, \xi), \bar{H}_{i 1}(m, \xi), \bar{H}_{i 2}(m, \xi)$ and $\bar{H}_{i 3}(m, \xi)$, takes the form

$$
\begin{aligned}
H_{i 0}(m, t)= & \sum_{k=0}^{\infty} \frac{(-1)^{k}\left(b_{i}\right)^{k}\left(\theta_{m}^{(i)}\right)^{2 k}}{\left(a_{i}\right)^{k+1}} \\
& \cdot G_{1-\gamma_{i},-(k+1) \gamma_{i}, k+1}\left(t,-\alpha a_{i}^{-1}\left(\theta_{m}^{i}\right)^{2}\right),
\end{aligned}
$$




$$
\begin{aligned}
& H_{i 3}(m, t)=\frac{\delta(t)}{a_{i}}-\frac{\alpha_{i}\left(\theta_{m}^{(i)}\right)^{2}}{a_{i}{ }^{2}} t^{1-\gamma_{i}} E_{1-\gamma_{i}, 1-\gamma_{i}}\left(\frac{-\alpha_{i}\left(\theta_{m}^{(i)}\right)^{2}}{a_{i}} t\right) \\
& +\sum_{k=0}^{\infty} \frac{(-1)^{k+1}\left(b_{i}\right)^{k+1}\left(\theta_{m}^{(i)}\right)^{2 k+2}}{\left(a_{i}\right)^{k+2}} \\
& \text { - } G_{1-\gamma_{i},-k \gamma_{i}, k+1}\left(t,-\alpha a_{i}^{-1}\left(\theta_{m}^{i}\right)^{2}\right) \text {, } \\
& H_{i 1}(m, t)=\sum_{k=0}^{\infty} \frac{(-1)^{k}\left(b_{i}\right)^{k+1}\left(\theta_{m}^{(i)}\right)^{2 k}}{\left(a_{i}\right)^{k+1}} \\
& \text { - } G_{1-\gamma_{i},-(k+1) \gamma_{i}, k+1}\left(t,-\alpha a_{i}^{-1}\left(\theta_{m}^{i}\right)^{2}\right) \\
& +\sum_{k=0}^{\infty} \frac{(-1)^{k}\left(b_{i}\right)^{k+1}\left(\theta_{m}^{(i)}\right)^{2 k}\left(\alpha_{i}\right)}{\left(a_{i}\right)^{k+1}} \\
& \text { - } G_{1-\gamma_{i},-k \gamma_{i}, k+1}\left(t,-\alpha a_{i}^{-1}\left(\theta_{m}^{i}\right)^{2}\right)
\end{aligned}
$$

and

$$
\begin{aligned}
u_{i}(y, t)= & \wp_{1 i}(y) u_{i}\left(d_{i-1}, t\right)+\wp_{2 i}(y) u_{i}\left(d_{i}, t\right) \\
& +\frac{2}{d_{i}-d_{i-1}} \sum_{m=1}^{\infty} \sin \left(\vartheta_{m}^{(i)}\left(y-d_{i-1}\right)\right) \frac{\left[1-(-1)^{m}\right]}{\vartheta_{m}^{(i)}} \cdot H_{i 0}(m, t) * P(t) \\
& -\frac{2}{d_{i}-d_{i-1}} \sum_{m=1}^{\infty} \sin \left(\vartheta_{m}^{(i)}\left(y-d_{i-1}\right)\right) \frac{a_{i}}{\vartheta_{m}^{(i)}} H_{i 3}(m, t) * u_{i}\left(d_{i-1}, t\right) \\
& +\frac{2}{d_{i}-d_{i-1}} \sum_{m=1}^{\infty} \sin \left(\vartheta_{m}^{(i)}\left(y-d_{i-1}\right)\right) \\
& \frac{a_{i}(-1)^{m}}{\vartheta_{m}^{(i)}} H_{i 3}(m, t) * u_{i}\left(d_{i-1}, t\right), \\
\vartheta_{m}^{(i)}= & \frac{m \pi}{d_{i}-d_{i-1}}, m=1,2, \ldots, i \in I_{n}^{1} .
\end{aligned}
$$

$$
\begin{aligned}
H_{i 2}(m, t)= & \frac{\delta(t)}{a_{i}}-\frac{\alpha_{i}\left(\theta_{m}^{(i)}\right)^{2}}{a_{i}^{2}} t^{1-\gamma_{i}} E_{1-\gamma_{i}, 1-\gamma_{i}}\left(\frac{-\alpha_{i}\left(\theta_{m}^{(i)}\right)^{2}}{a_{i}} t\right) \\
& +\sum_{k=0}^{\infty} \frac{(-1)^{k+1}\left(b_{i}\right)^{k+1}\left(\theta_{m}^{(i)}\right)^{2 k+2}}{\left(a_{i}\right)^{k+2}} \\
& \cdot G_{1-\gamma_{i}-(k+2) \gamma_{i}+1, k+2}\left(t,-\alpha a_{i}^{-1}\left(\theta_{m}^{i}\right)^{2}\right) \\
& +\frac{\alpha_{i}}{a_{i}} \delta^{\prime}(t) * t^{1-\gamma_{i}} E_{1-\gamma_{i}, 1-\gamma_{i}}\left(\frac{-\alpha_{i}\left(\theta_{m}^{(i)}\right)^{2}}{a_{i}} t\right) \\
& +\sum_{k=0}^{\infty} \frac{(-1)^{k+1}\left(b_{i}\right)^{k+1}\left(\theta_{m}^{(i)}\right)^{2 k+2}\left(\alpha_{i}\right)}{\left(a_{i}\right)^{k+2}} \\
& \cdot G_{1-\gamma_{i}-\left((k+1) \gamma_{i}-1\right), k+2}\left(t,-\alpha a_{i}^{-1}\left(\theta_{m}^{i}\right)^{2}\right)
\end{aligned}
$$

where $h_{1}(t) * h_{2}(t)=\int_{0}^{1} h_{1}(t-\tau) h_{2}(\tau) d \tau$ denotes the convolution product of the functions $h_{1}(t), h_{2}(t)$. Using Eqs. (26), (43) and (44), we obtain for velocities the following expressions:

The shear stresses system $\tau_{i}(y, \xi), i \in I_{n^{\prime}}^{1}$ can be obtained by applying inverse Laplace transform to Eqs. (27), (28) and using Eqs. (45), (46), we get

$$
\begin{aligned}
\tau_{i}(y, t)= & T_{i 1}(y, t) * u_{i}\left(d_{i}, t\right)-T_{i 2}(y, t) * u_{i}\left(d_{i-1}, t\right) \\
& +T_{i 3}(y, t) * P(t),
\end{aligned}
$$

where 


$$
\begin{array}{rlrl}
T_{i 1}(y, t)= & {\left[\frac{1}{d_{i}-d_{i-1}}\left(\alpha_{i} t^{\alpha_{i}-1} E_{\alpha_{i}, \alpha_{i}}\left(-b_{i} \alpha_{i}^{-1} t^{\alpha_{i}}\right)\right)\right.} & \bar{\tau}_{i}(y, \xi)= & \left(b_{i}+\alpha_{i} \xi^{\gamma_{i}}\right) \frac{\partial \bar{u}_{i}(y, \xi)}{\partial y} \\
& \left.+2 \sum_{m=1}^{\infty} a_{i}(-1)^{m} H_{i 2}(m, t) \cos \left(\vartheta_{m}^{(i)}\left(y-d_{i-1}\right)\right)\right], & =\left(b_{i}+\alpha_{i} \xi^{\gamma_{i}}\right)\left[-\sqrt{m_{i}(\xi)} A_{i}(\xi) e^{-y \sqrt{m_{i}(\xi)}}\right. \\
& \left.+\sqrt{m_{i}(\xi)} B_{i}(\xi) e^{y \sqrt{m_{i}(\xi)}}\right]
\end{array}
$$$$
T_{i 2}(y, t)=\left[\frac{1}{d_{i}-d_{i-1}}\left(\alpha_{i} t^{\alpha_{i}-1} E_{\alpha_{i}, \alpha_{i}}\left(-b_{i} \alpha_{i}^{-1} t^{\alpha_{i}}\right)\right)\right.
$$$$
\left.+2 \sum_{m=1}^{\infty} a_{i} H_{i 2}(m, t) \cos \left(\vartheta_{m}^{(i)}\left(y-d_{i-1}\right)\right)\right] \text {, }
$$

Using the boundary conditions (18) and the interface conditions (19), we have

$C_{1}(\xi) D_{1}(\xi)=E_{1}(\xi)$,

$T_{i 3}(y, t)=\frac{2}{d_{i}-d_{i-1}} \sum_{m=1}^{\infty}\left[1-(-1)^{m}\right] H_{i 1}(m, t) \cos \left(\vartheta_{m}^{(i)}\left(y-d_{i-1}\right)\right), i \in I_{n}^{1}$.

The considered model of multi-layer flow of second grade fluids is more general and several special cases can be deduced from it. In order to certify the correctness of results that have been here obtained, as well as to get some physical insight of them, we consider a particular case whose solution is well known in the existing literature or can be easily determined. For the special case when $\alpha_{i} \rightarrow 0$ in Eqs. (47) and (48), we obtain analytical solutions of velocities for the $n$-immiscible Newtonian fluids. These velocities (47) of $n$-immiscible fluids match with the results obtained in ([16]; Eq. (72) with $\lambda_{i} \rightarrow 0$ ).

\subsection{Semi-analytical solution for velocity and shear stress}

In this subsection, we obtained a new solution for the velocities and shear stresses corresponding to the governing equations given by Eqs. (16)-(19) by applying the Laplace transform in conjunction with the classical method for the ordinary differential equations. The general solution for the Eq. (20) provides a system of the Laplace transformed velocities as

$\bar{u}_{i}(y, \xi)=A_{i}(\xi) e^{-y \sqrt{m_{i}(\xi)}}+B_{i}(\xi) e^{y \sqrt{m_{i}(\xi)}}+\frac{\bar{P}(\xi)}{a_{i} \xi}$,

where

$m_{i}(\xi)=\frac{a_{i} \xi}{\left(b_{i}+\alpha_{i} \xi^{\gamma_{i}}\right)}, i \in I_{n}^{1}$.

Now with Eqs. (17) and (50), the Laplace transformed form of the shear stresses $\tau_{i}(y, t), i \in I_{n^{\prime}}^{1}$, can be written as where the $C_{1}(\xi)$ is given by

$C_{1}(\xi)=\left[\begin{array}{cccccccccc}1 & 0 & 0 & \cdots & 0 & 1 & 0 & 0 & \cdots & 0 \\ \mathfrak{f}_{1} & -\mathfrak{g}_{1} & 0 & \cdots & 0 & \frac{1}{\mathfrak{f}_{1}} & -\frac{1}{\mathfrak{g}_{1}} & 0 & \cdots & 0 \\ 0 & \mathfrak{f}_{2} & -\mathfrak{g}_{2} & \cdots & 0 & 0 & \frac{1}{\mathfrak{f}_{2}} & -\frac{1}{\mathfrak{g}_{2}} & \cdots & 0 \\ \vdots & \vdots & \ddots & \ddots & \vdots & \vdots & \vdots & \vdots & \ddots & \vdots \\ 0 & 0 & \cdots & \mathfrak{f}_{n-1} & -\mathfrak{g}_{n-1} & 0 & 0 & 0 & \frac{1}{\mathfrak{f}_{n-1}} & -\frac{1}{\mathfrak{g}_{n-1}} \\ \mathfrak{h}_{1} & -\mathfrak{f}_{1} & 0 & \cdots & 0 & \mathfrak{l}_{1} & -\mathfrak{p}_{1} & 0 & \cdots & 0 \\ 0 & \mathfrak{h}_{2} & -\mathfrak{f}_{2} & \cdots & 0 & 0 & \mathfrak{l}_{2} & -\mathfrak{p}_{2} & \cdots & 0 \\ \vdots & \vdots & \ddots & \cdots & \vdots & \vdots & \vdots & \ddots & \ddots & \vdots \\ 0 & 0 & \cdots & \mathfrak{h}_{n-1} & -\mathfrak{f}_{n-1} & 0 & 0 & 0 & \mathfrak{l}_{n-1} & -\mathfrak{p}_{n-1} \\ 0 & 0 & \cdots & 0 & \mathfrak{h}_{n} & 0 & 0 & 0 & 0 & \mathfrak{l}_{n}\end{array}\right]$

and the columns $D_{1}(\xi)$ and $E_{1}(\xi)$ are

$D_{1}(\xi)=\left[\begin{array}{c}A_{1}(\xi) \\ A_{2}(\xi) \\ \vdots \\ A_{n}(\xi) \\ B_{1}(\xi) \\ B_{2}(\xi) \\ \vdots \\ B_{n}(\xi)\end{array}\right], E_{1}(\xi)=\left[\begin{array}{c}P_{0}(\xi) \\ P_{1}(\xi) \\ \vdots \\ P_{n-1}(\xi) \\ 0 \\ 0 \\ \vdots \\ 0\end{array}\right]$

here 


$$
\begin{aligned}
\mathfrak{f}_{j} & =e^{-d_{j} \sqrt{m_{j}(\xi)}}, \mathfrak{g}_{j}=e^{-d_{j} \sqrt{m_{j+1}(\xi)}}, \\
\mathfrak{h}_{j} & =-\sqrt{m_{j}(\xi)} e^{-d_{j} \sqrt{m_{j}(\xi)}}, \\
\mathfrak{f}_{j} & =-\left(\frac{b_{j+1}+\alpha_{j+1} \xi^{\gamma_{j+1}}}{b_{j}+\alpha_{j} \xi^{\gamma_{j}}}\right) \sqrt{m_{j+1}(\xi)} e^{-d_{j}} \sqrt{m_{j+1}(\xi)}, \\
\mathfrak{l}_{j} & =-\sqrt{m_{j}(\xi)} e^{d_{j}} \sqrt{m_{j}(\xi)}, \\
\mathfrak{p}_{j} & =\left(\frac{b_{j+1}+\alpha_{j+1} \xi^{\gamma_{j+1}}}{b_{j}+\alpha_{j} \xi^{\xi_{j}}}\right) \sqrt{m_{j+1}(\xi)} e^{d_{j}} \sqrt{m_{j+1}(\xi)}, \\
P_{0}(\xi) & =\bar{f}(\xi)-\frac{\bar{P}(\xi)}{a_{1} \xi}, \\
P_{j}(\xi) & =\left(\frac{1}{a_{j+1}}-\frac{1}{a_{j}}\right) \frac{\bar{P}(\xi)}{\xi}, j=1,2, \ldots, n-1
\end{aligned}
$$

The system (52) can be written in equivalent form as,

$$
\begin{aligned}
W_{1}(\xi) A(\xi)+X_{1}(\xi) B(\xi) & =L_{3}(\xi), \\
Y_{1}(\xi) A(\xi)+Z_{1}(\xi) B(\xi) & =L_{4}(\xi),
\end{aligned}
$$

Where

$$
\begin{aligned}
& W_{1}=\left(W_{i j}^{\prime}\right)_{i, j \in I_{n-1}^{0}} \vdots X_{1}=\left(X_{i j}^{\prime}\right)_{i, j \in I_{n-1}^{0}} \\
& W_{0 j}^{1}=\delta_{0, j}, j \in l_{n-1}^{0}: X_{0 j}^{1}=\delta_{0, j}, j \in l_{n-1}^{0} \\
& W_{i j}^{\prime}=\mathfrak{f}_{i} \delta_{i, j+1}-\mathfrak{g}_{i} \delta_{i+1, j+1}, \quad X_{i j}^{1}=\frac{1}{\mathfrak{f}_{i}} \delta_{i, j+1}-\frac{1}{\mathfrak{g}_{i}} \delta_{i+1, j+1}, \\
& i \in I_{n-1}^{1}, j \in I_{n-1}^{0}, \vdots i \in I_{n-1}^{1}, j \in I_{n-1}^{0} \text {, } \\
& Y_{1}=\left(Y^{\prime}{ }_{i j}\right)_{i,\left.j \in\right|_{n-1} ^{0}} \vdots Z_{1}=\left(Z^{\prime}{ }_{i j}\right)_{i,\left.j \in\right|_{n-1} ^{0}} \\
& Y_{i j}^{\prime}=\mathfrak{h}_{i+1} \delta_{i+1, j+1}-\mathfrak{l}_{i+1} \delta_{i+2, j+1,}: Z_{i j}^{\prime}=\mathfrak{l}_{i+1} \delta_{i+1, j+1}-\mathfrak{p}_{i+1} \delta_{i+2, j+1} \text {, } \\
& i \in l_{n-2^{\prime}}^{0} j \in l_{n-1}^{0}, \vdots i \in l_{n-2^{\prime}}^{0} j \in l_{n-1}^{0} \text {, } \\
& Y_{(n-1) j}^{\prime}=\mathfrak{h}_{n} \delta_{n-1, j} j \in I_{n-1}^{0} \vdots Z_{(n-1) j}^{\prime}=\mathfrak{l}_{n} \delta_{n-1, j} j \in l_{n-1}^{0}, \\
& L_{3}=\left(\begin{array}{c}
P_{0}(\xi) \\
P_{1}(\xi) \\
\vdots \\
P_{n-1}(\xi)
\end{array}\right), L_{4}=\left(\begin{array}{c}
0 \\
0 \\
\vdots \\
0
\end{array}\right)_{n \times 1} \text {, } \\
& A=\left(\begin{array}{c}
A_{1}(\xi) \\
A_{2}(\xi) \\
\vdots \\
A_{n}(\xi)
\end{array}\right), B=\left(\begin{array}{c}
B_{1}(\xi) \\
B_{2}(\xi) \\
\vdots \\
B_{n}(\xi)
\end{array}\right)
\end{aligned}
$$

Where $\delta_{i j}$ is the Kronecker tensor. Matrices $W_{1}, X_{1}, Y_{1}, Z_{1}$ are invertible triangular matrices, therefore Eqs. (47) can be written as

$$
\begin{aligned}
\left(X_{1}^{-1} W_{1}-Z_{1}^{-1} Y_{1}\right) A & =X_{1}^{-1} L_{3} \\
B & =-Z_{1}^{-1} Y_{1} A
\end{aligned}
$$

Suppose that matrix $S_{1}=X_{1}^{-1} W_{1}-Z_{1}^{-1} Y_{1}$ is invertible, we have

$$
\begin{aligned}
& A=S_{1}^{-1} X_{1}^{-1} L_{3} \\
& B=-Z_{1}^{-1} Y_{1} S_{1}^{-1} X_{1}^{-1} L_{3}
\end{aligned}
$$

where

$$
\begin{aligned}
& W_{1}^{-1}=\left(w_{i j}^{1}\right) i, j \in I_{n-1}^{0}, \\
& w_{0 j}^{1}=\delta_{0 j} j \in I_{n-1}^{0}, w_{i 0}^{1}=\prod_{r=1}^{i} \frac{\mathfrak{f}_{r}}{\mathfrak{g}_{r}}, i \in l_{n-1}^{1}, \\
& w_{i j}^{1}=-\prod_{s=1}^{i} \frac{\mathfrak{f}_{s}}{\mathfrak{g}_{s}} \prod_{r=0}^{j-1} \frac{\mathfrak{g}_{r}}{\mathfrak{f}_{r+1}} \sum_{k=0}^{n-1} \delta_{i, j+k} i, j \in I_{n-1}^{1} ; \\
& X_{1}^{-1}=\left(x_{i j}^{1}\right) i, j \in I_{n-1}^{0} \text {, } \\
& x_{0 j}^{1}=\delta_{0 j}, j \in I_{n-1}^{0}, x_{i 0}^{1}=\prod_{r=1}^{i} \frac{\mathfrak{f}_{r}}{\mathfrak{g}_{r}}, i \in I_{n-1}^{1}, \\
& x_{i j}^{1}=-\prod_{s=1}^{i} \frac{\mathfrak{f}_{s}}{\mathfrak{g}_{s}} \prod_{r=0}^{j-1} \frac{\mathfrak{f}_{r+1}}{\mathfrak{g}_{r}} \sum_{k=0}^{n-1} \delta_{i, j+k}, i, j \in I_{n-1}^{1} ; \\
& z_{1}^{-1}=\left(z_{i j}^{1}\right)_{i, j \in l_{n-1}^{0}} \\
& \text { with, } \\
& z_{i j}^{1}=\left(\prod_{s=0}^{i} \frac{\mathfrak{l}_{s}}{\mathfrak{p}_{s}} \prod_{r=0} \frac{\mathfrak{p}_{s}}{\mathfrak{l}_{r+1}}\right) \sum_{k=0}^{n-1} \delta_{i-k, j^{\prime}} \mathfrak{l}_{0}=\mathfrak{p}_{0}=\mathfrak{l}_{n}=1, i, j \in I_{n-1}^{0} .
\end{aligned}
$$

And the matrix $S_{1}=\left(S_{i j}^{1}\right)_{i,\left.j \in\right|_{n-1} ^{0}}$ is defined by the elements

$S_{i j}^{1}=\sum_{k=0}^{n-1}\left(x_{i k}^{1} W_{k j}^{1}-z_{i k}^{1} Y_{k j}^{1}\right)$

The Laplace transforms given by Eqs. (50), (51) and (55) involve intricate functions, which makes it difficult to apply analytical methods to compute the inverse Laplace transforms of these relations.

Here we have used two precision numerical procedures for the Laplace inversion of Eqs. (50),(51) and (55), specifically the fixed Talbot procedure and the improved Talbot procedure $[37,38]$.

Consider a function $g(y, t)$ with the Laplace transform $\bar{G}(y, s)$. Talbot algorithm [37] approximates the function $g(y, t)$ from a given function $\bar{G}(y, s)$ as

$$
\begin{aligned}
g(y, t) \cong & \frac{r}{M}\left\{\frac{\exp (r t)}{2} \bar{G}(y, r)\right. \\
& \left.+\sum_{k=1}^{M-1} \operatorname{Re}\left[\exp \left(t z\left(\Omega_{k}\right)\right) \bar{G}\left(y, z\left(\Omega_{k}\right)\right)\left(1+i \zeta\left(\Omega_{k}\right)\right)\right]\right\}
\end{aligned}
$$

where 


$$
\begin{aligned}
r & =\frac{2 M}{5 t}, \quad z(\Omega)=r \Omega(\cot \Omega+i), \quad \Omega \in(-\pi, \pi), \\
\zeta(\Omega) & =\Omega+(\Omega \cot \Omega-1) \cot \Omega, \Omega_{k}=\frac{k \pi}{M} .
\end{aligned}
$$

The improved Talbot algorithm is applied to approximate the function $g(y, t)$ as

$$
g(y, t) \cong \sum_{k=1}^{M} \bar{G}\left(y, z_{1}\left(\sigma_{k}\right)\right)\left(v+i \zeta_{1}\left(\sigma_{k}\right)\right) \frac{\exp \left(t z_{1}\left(\sigma_{k}\right)\right)}{t}
$$

where,

$$
\begin{aligned}
& z_{1}(\Omega)=\frac{M}{t}[v i \Omega+\mu \Omega \cot (\alpha \Omega)-\Omega], \quad \Omega \in[-\pi, \pi] \\
& \zeta_{1}(\Omega)=\alpha \mu \Omega+\mu(\alpha \Omega \cot (\alpha \Omega)-1) \cot (\alpha \Omega), \sigma_{k}=\frac{(2 k-1) \pi}{M}-\pi .
\end{aligned}
$$

Here the parameters $M, \alpha, \mu, v, \xi$ are quantified by the handler.

The results obtained by Luo et al. [39], are the special case $n=2$, of our more general semi-analytical solutions (50)-(51).
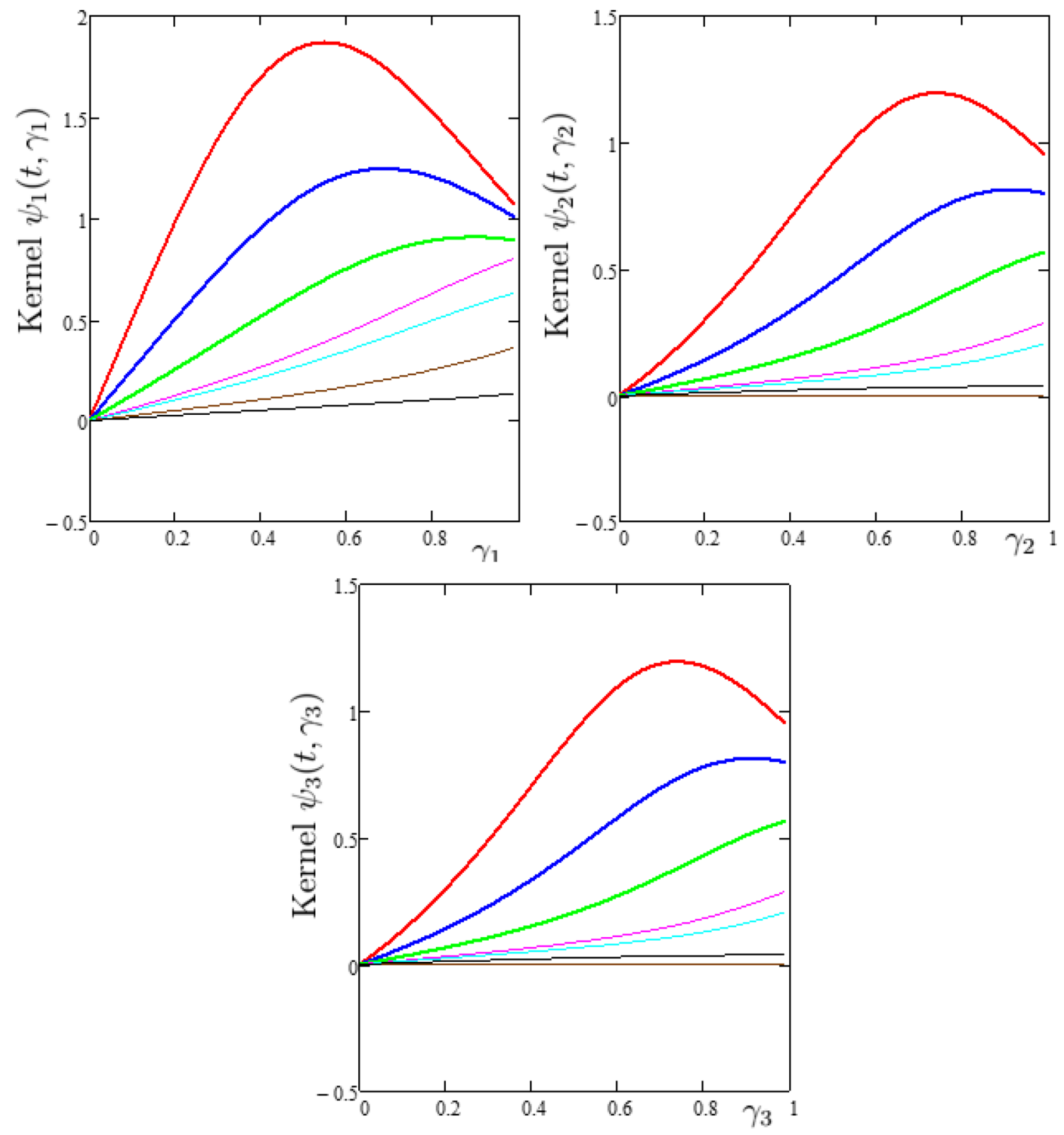

Fig. 2 Variation of the damping kernels $\psi_{i}\left(t, \gamma_{i}\right), i=1,2,3$, with the fractional parameters $\gamma_{i}, i=1,2,3$ 


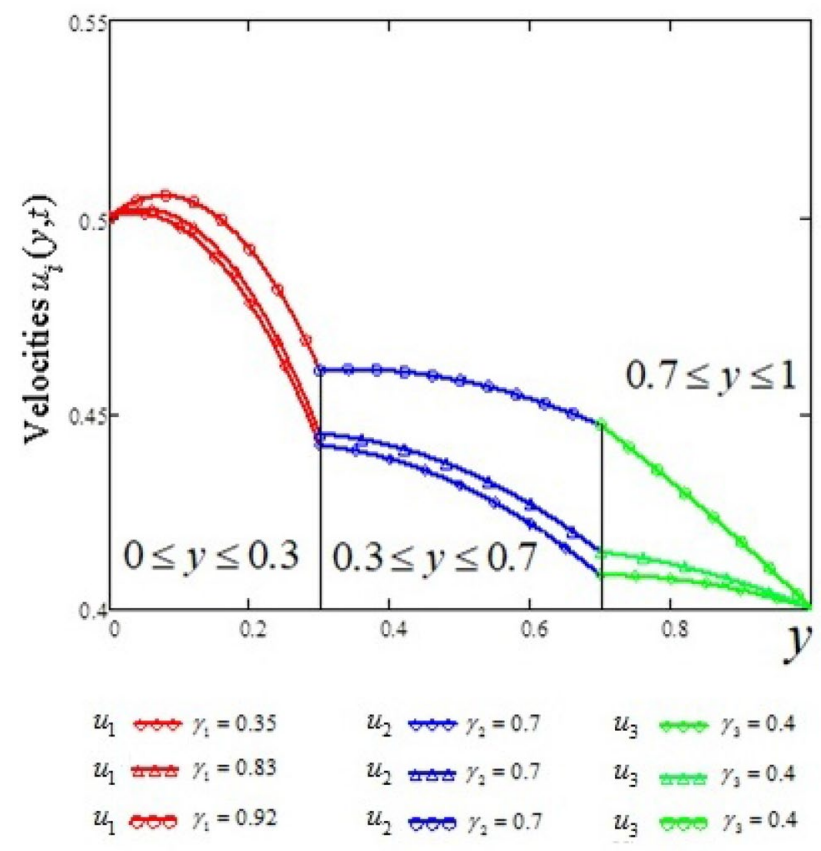

Fig. 3 Profile of velocities $u_{i}(y, t), \quad i=1,2,3$, at $\mu_{1}=0.05, \mu_{2}=0.3, \mu_{3}=0.5, \alpha_{1}=0.2, \alpha_{2}=0.3, \alpha_{3}=0.6$ and for different values of $\gamma_{1}$

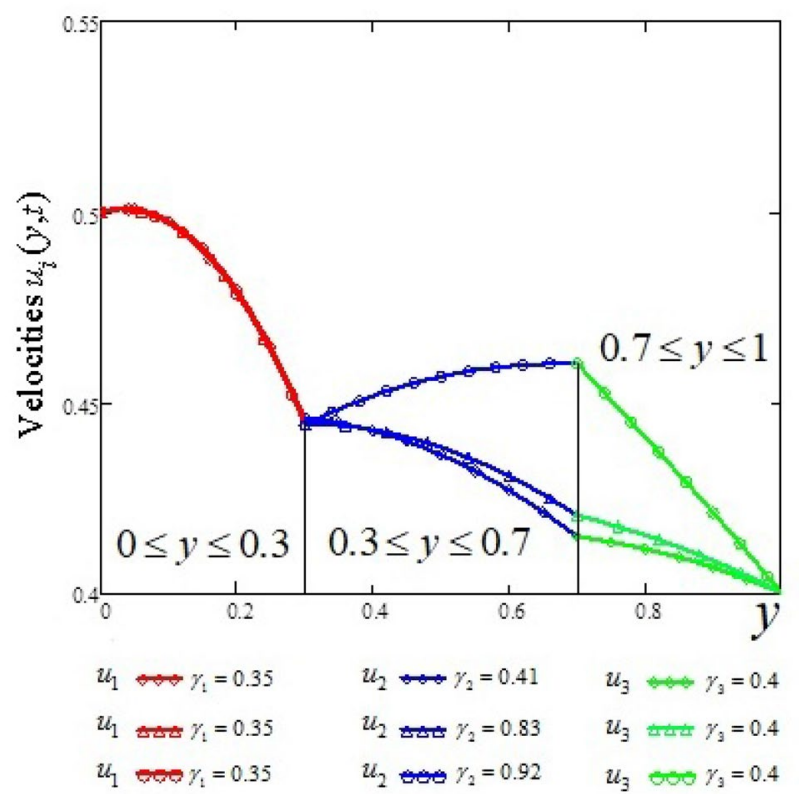

Fig. 4 Profile of velocities $u_{i}(y, t), \quad i=1,2,3$, at $\mu_{1}=0.05, \mu_{2}=0.3, \mu_{3}=0.5, \alpha_{1}=0.2, \alpha_{2}=0.3, \alpha_{3}=0.6$ and for different values of $\gamma_{2}$

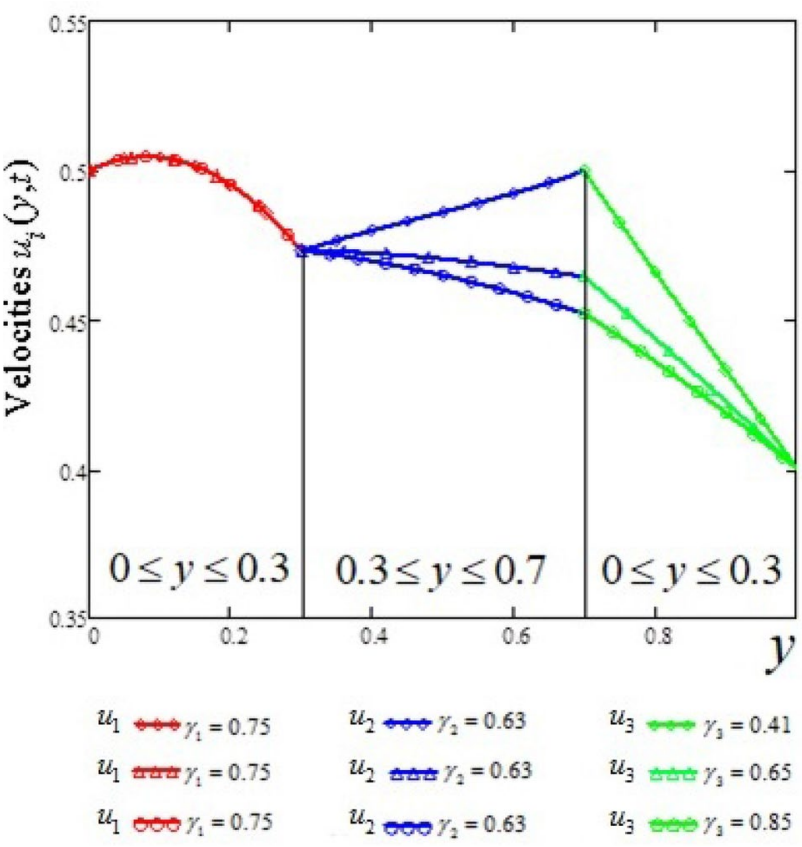

Fig. 5 Profile of velocities $u_{i}(y, t), \quad i=1,2,3$, at $\mu_{1}=0.05, \mu_{2}=0.3, \mu_{3}=0.5, \alpha_{1}=0.2, \alpha_{2}=0.3, \alpha_{3}=0.6$ and for different values of $\gamma_{3}$

\section{Numerical results and discussions}

The unsteady, laminar flow of multi-layer fractional n-immiscible second-grade fluids between two parallel plates have been investigated. The flow of the fluids is caused by the time-dependent pressure gradient along the axis of the flow and by the movement of the channel walls in their planes with the time-dependent velocities, and in the presence of the fluid-fluid interfacial conditions.

A generalized mathematical model based on the fractional differential constitutive equation with time-fractional Caputo derivative has been developed and studied. On the solid boundaries, the no-slip condition is considered, while at the fluid-fluid interfaces, the velocity and shear stress are considered continuous.

Semi-analytical solutions of the problem with initial, boundary and interface conditions have been determined by employing the Laplace transform coupled with the Talbot algorithms for the numerical inverse Laplace transforms. Using the Laplace transform and the finite sine-Fourier transform, the analytical solutions of the same problem have been determined.

In the present study, we considered the particular case of three layers of immiscible fluids when the velocity of the bottom plate is $u_{1}(0, t)=f(t)=0.5 H(t)$ the velocity of the upper plate is $u_{3}(0, t)=g(t)=0.4 H(t)$. $H(t)=\left\{\begin{array}{l}0, t \leq 0 \\ 1, t>0\end{array}=\frac{1}{2} \operatorname{sign}(t)(1+\operatorname{sign}(t))\right.$ is the unit step 
Fig. 6 Profile of velocities $u_{i}(y, t), i=1,2,3$, at $\gamma_{1}=0.8, \gamma_{2}=0.7, \gamma_{3}=0.4$ $\alpha_{1}=0.2, \alpha_{2}=0.3, \alpha_{3}=0.6$ and for different values of $\mu_{1}$

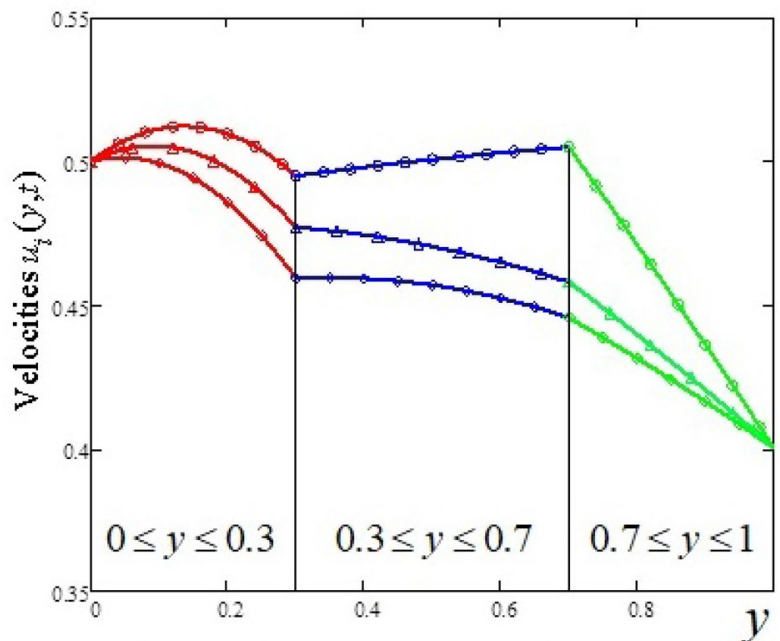

$\begin{array}{lll}u_{1} \leftrightarrow \mu_{1}=0.05 & u_{2} \Leftrightarrow \mu_{2}=0.26 & u_{3} \Leftrightarrow \mu_{3}=0.6 \\ u_{1} \mu_{1}=0.05 & u_{2} \mu_{2}=0.41 & u_{3} \\ u_{1} \mu_{1}=0.05 & u_{2} \leftrightarrow \mu_{2}=0.6\end{array}$

Fig. 7 Profile of velocities $u_{i}(y, t), \quad i=1,2,3$, at $\gamma_{1}=0.8, \gamma_{2}=0.7, \gamma_{3}=0.4, \alpha_{1}=0.2, \alpha_{2}=0.3, \alpha_{3}=0.6$ and for different values of $\mu_{2}$

Heaviside function, and the pressure gradient is $P(t)=1+\sin (t)$. The flows regions are determined by $d_{0}=0, d_{1}=0.3, d_{2}=0.7, d_{3}=1$.

With the help of Math-cad software, numerical results have been obtained and graphically presented for the velocity of the fluids. Some aspects of the fluid motion are presented in Figs. 2, 3, 4, 5, 6, 7, 8, 9 and 10 that are sketched for the following values of the densities of
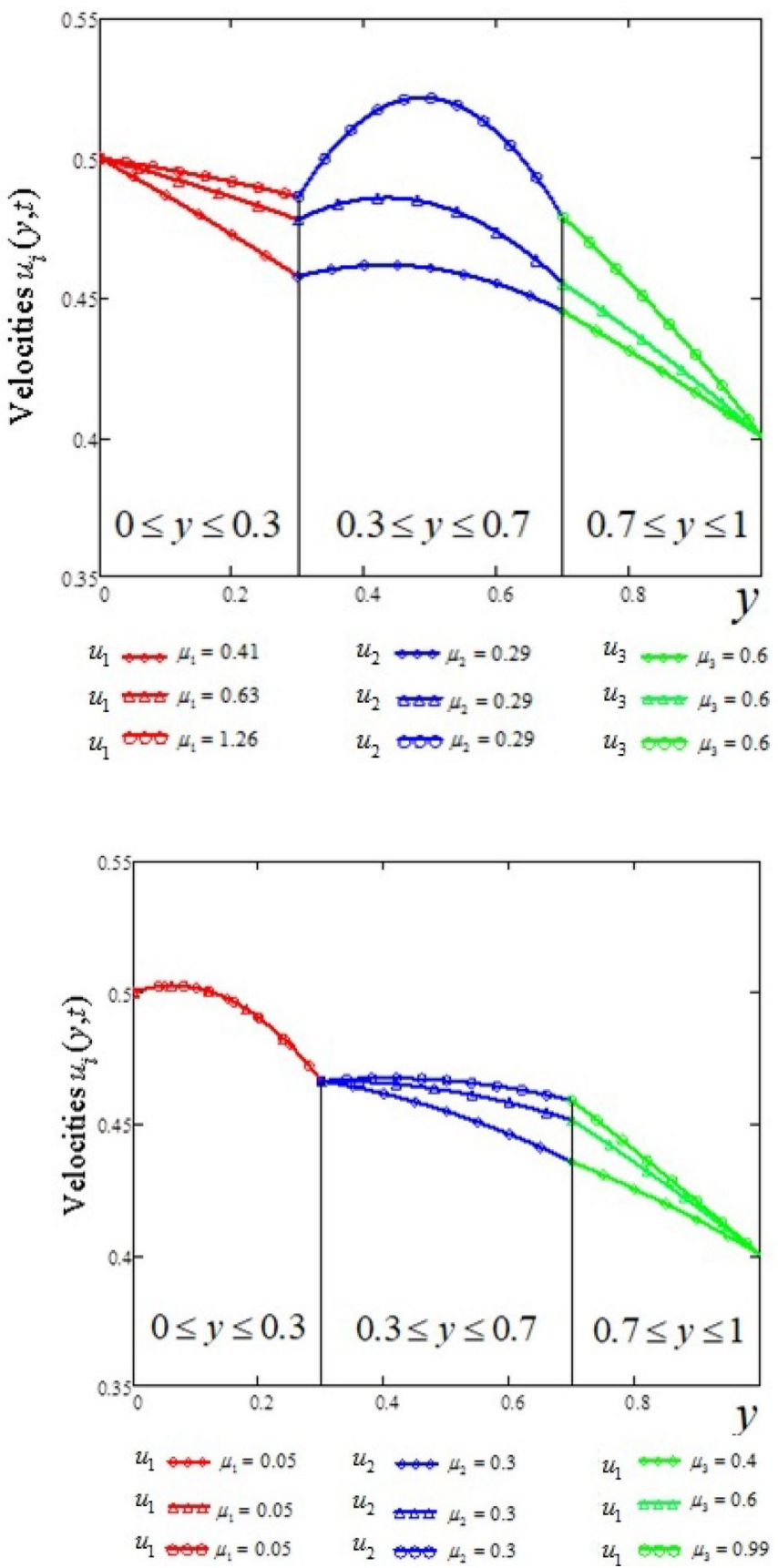

Fig. 8 Profile of velocities $u_{i}(y, t), \quad i=1,2,3$, at $\gamma_{1}=0.8, \gamma_{2}=0.7, \gamma_{3}=0.4, \alpha_{1}=0.2, \alpha_{2}=0.3, \alpha_{3}=0.6$ and for different values of $\mu_{3}$

fluids $\rho_{1}=1000, \rho_{2}=1300, \rho_{3}=1500$ (other parameters are given in the figures' labels).

To emphasize the influence of the memory on the fluid motion, let's refer to the constitutive Eq. (11) that can be written in the equivalent form $\frac{\partial u(y, t)}{\partial y}=\frac{1}{\alpha_{i}} \int_{0}^{t} E_{\gamma_{i}, \gamma_{i}}\left(-\frac{b_{i}}{\alpha_{i}}(t-s)^{\gamma_{i}}\right) \tau_{i}(y, s) d s$. This relationship shows that the shear rate is strongly influenced by the 


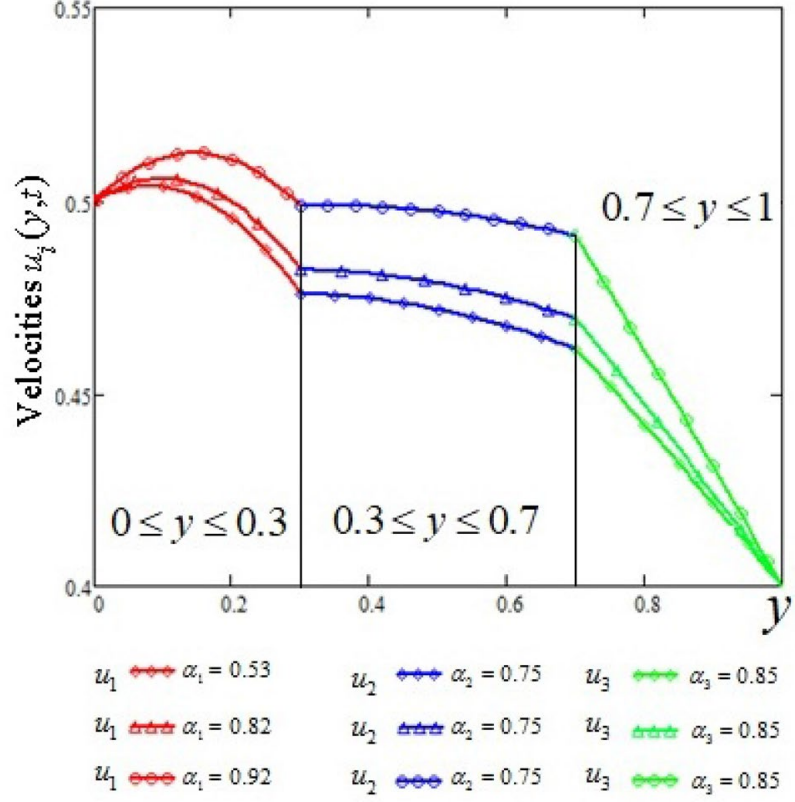

Fig. 9 Profile of velocities $u_{i}(y, t), \quad i=1,2,3$, at $\gamma_{1}=0.8, \gamma_{2}=0.7, \gamma_{3}=0.4, \mu_{1}=0.05, \mu_{2}=0.3, \mu_{3}=0.5$ and for different values of $\alpha_{1}$

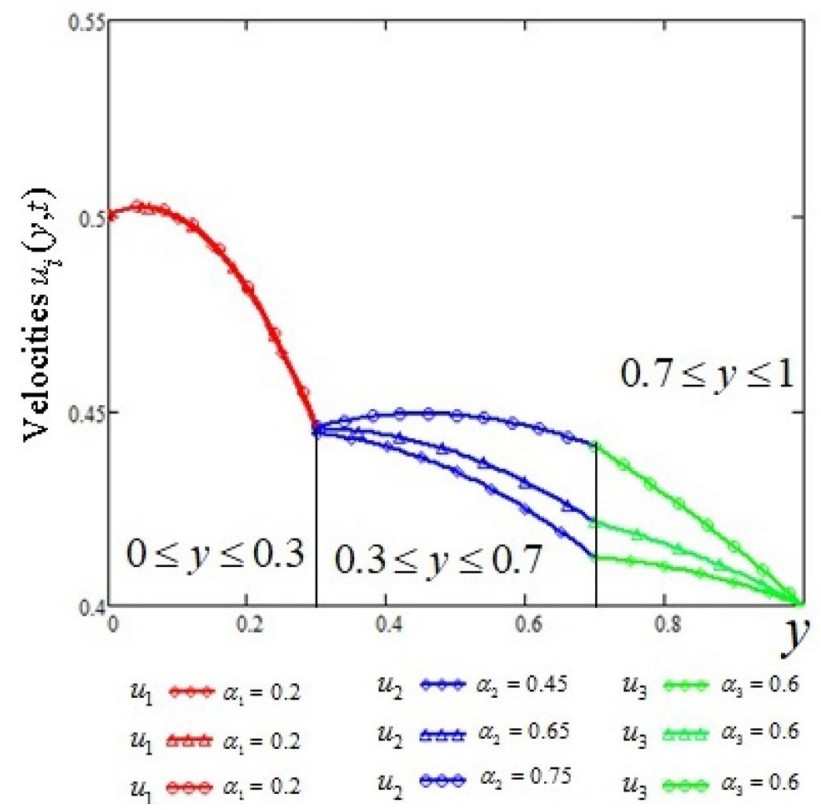

Fig. 10 Profile of velocities $u_{i}(y, t), \quad i=1,2,3$, at $\gamma_{1}=0.8, \gamma_{2}=0.7, \gamma_{3}=0.4, \mu_{1}=0.05, \mu_{2}=0.3, \mu_{3}=0.5$ and for different values of $\alpha_{2}$

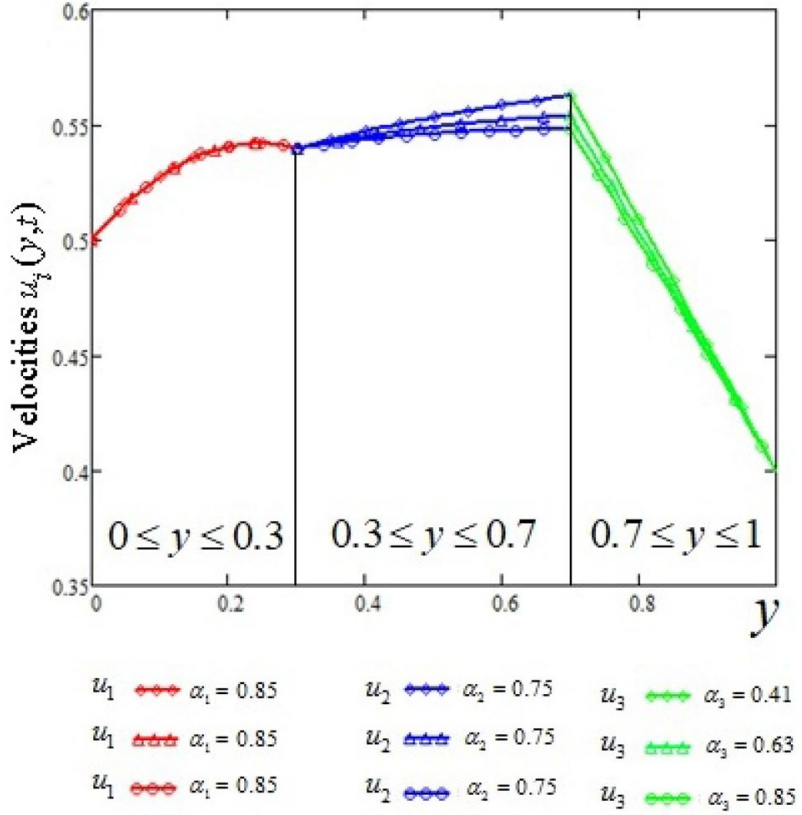

Fig. 11 Profile of velocities $u_{i}(y, t), \quad i=1,2,3$, at $\gamma_{1}=0.8, \gamma_{2}=0.7, \gamma_{3}=0.4, \mu_{1}=0.05, \mu_{2}=0.3, \mu_{3}=0.5$ and for different values of $\alpha_{3}$

memory kernel present in the mathematical model, namely, by the weight function $E_{\gamma_{i} \gamma_{i}}\left(-\frac{b_{i}}{\alpha_{i}} t \gamma_{i}\right)$. The profiles of kernels corresponding to three fluids considered in the present study are given in Fig. 2, for different values of the time $\mathrm{t}, t \in\{0.05,0.1,0.2,0.4,0.5,0.8,1\}$. Note that, in the case of the ordinary fluids $\left(\gamma_{i}=1\right)$ fluids defined by the constitutive equation with the derivative of integer order, the dumping kernel is the exponential function $\exp \left(\frac{-b_{i} t}{\alpha_{i}}\right)$ has a profile different from the profiles shown in Fig. 2. It is observed from Fig. 2 that for small values of time $t$, the damping fractional kernel increases to a maximum value and then decreases to a minimum value. For time values $t$ greater than or equal to 0.4 , the kernel values are ascending by the fractional parameter. On the other hand, for positive values of the argument, the exponential function $\exp \left(\frac{-b_{i} t}{\alpha_{i}}\right)$ is decreasing.This discussion highlights that there are essential differences between memory effects for the fractional and ordinary fluids. The different behavior of the dumping kernel for the shear rate in the case of the fractional model, respectively for the ordinary model, it is visible to the profiles of the velocities of fluids presented in Figs. 3, 4, 5, 6, 7, 8, 9, 10 and 11.

Figure 2 has been plotted to study the influence of the memory of the fluid in the first layer on the fluids in layers two and three which are considered with constant fractional parameters. It is seen in Fig. 2 that for increasing the 


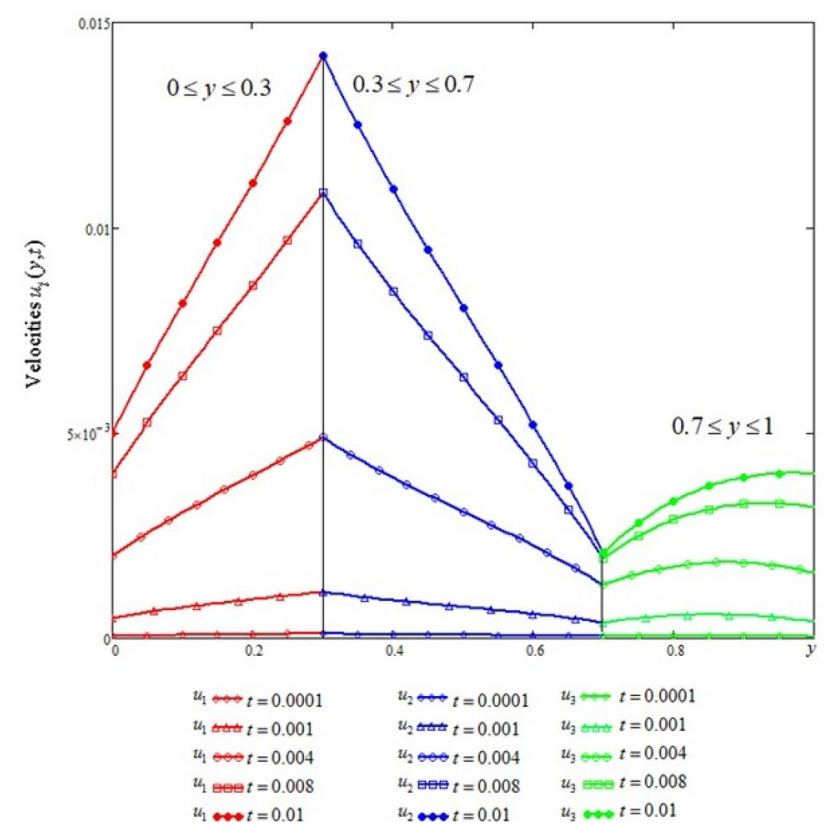

Fig. 12 Profile of velocities $u_{i}(y, t), \quad i=1,2,3$, at $\mu_{1}=0.05, \mu_{2}=0.3, \mu_{3}=0.4, \alpha_{1}=0.2, \alpha_{2}=0.4, \alpha_{3}=0.6, \gamma_{1}=0.8$, $\gamma_{2}=0.7, \gamma_{3}=0.4$ and for different values of $t$

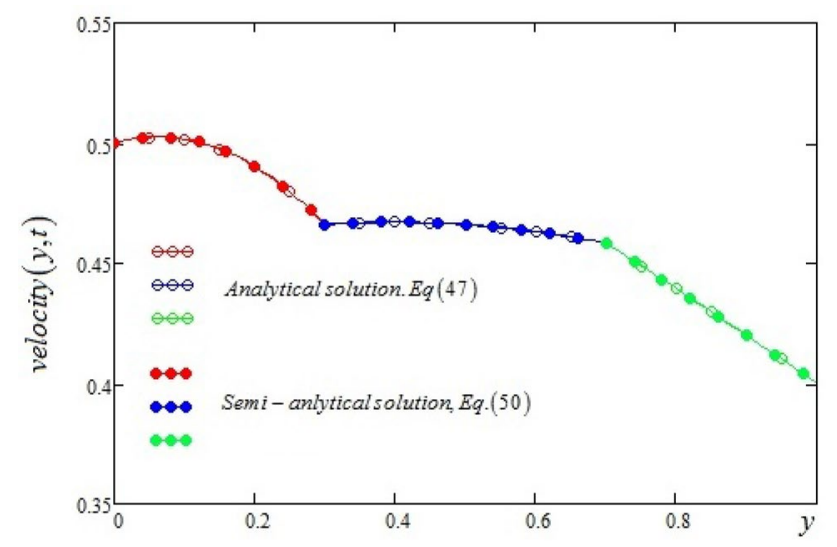

Fig. 13 Comparison between results obtained with analytical and semi-analytical solutions for velocities $u_{i}(y, t), i=1,2,3$

values of the fractional parameter in the first fluid layer, its velocity decreases. Obviously, the motion of the first layer influences the second and third layers through the interface interactions.

Figures 3, 4 and 5 present the influence of the variation of the fractional parameters in layers two and three. We must point out that in these cases the effect on the velocity of the fluid in the first layer is null. This was to be expected because there are no changing parameters in the first layer, so the speed on the interface between the first layer and the second layer will be the same due to the imposed continuity conditions.
The influence of the dynamic viscosity of the fluids on their velocity is analyzed in Figs. 6, 7 and 8. Taking into account the mathematical model, the influence of the dynamic viscosity occurs through the parameters $b_{i}$.

The variation of these parameters changes the kernel's profile of the fractional/integer derivative, so the shape of the velocity field. As expected and as shown in Fig. 6 , the fluid with the lowest viscosity has the highest velocity because, in this case, the viscous forces have lower values.

The influence of the material coefficients specific of the second-grade fluids, namely $\alpha_{i}$, on the fluid motion, was investigated in Figs. 9, 10 and 11. Like the dynamic viscosity, the material coefficients $\alpha_{i}$ are in the expression of the fractional derivative kernels, so they participate significantly in the memory process.

In Fig. 12 is presented the profile of velocities $u_{i}(y, t)$, $i=1,2,3$, for different values of the time $t$. It can be observed from Fig. 12 that at a very short time the profile for velocities $u_{i}(y, t), i=1,2,3$, are similar to the initial condition, that is zero everywhere.

In order to verify the accuracy of numerical results, we used the analytical solution of velocity given by Eq. (47) and the semi-analytical solution given by Eq. (50) for following values of parameters: $\mu_{1}=0.05, \mu_{2}=0.3, \mu_{3}=0.95$, $\alpha_{1}=0.2, \alpha_{2}=0.4, \alpha_{3}=0.6, \gamma_{1}=0.8, \gamma_{2}=0.7, \gamma_{3}=0.4$ and $t=3$. Numerical results are given in Fig. 13. There is a good agreement between the results obtained with the two formulas.

\section{Conclusion}

The simultaneous flow of generalized n-immiscible second grade fluids with the Caputo time-fractional derivative have been studied between two parallel plates. The unsteady, laminar simultaneous $\mathrm{n}$-fluids flow is caused by the motion of the side walls with time dependent velocities, and by the time dependent pressure gradient in flow direction.

On the solid boundaries, the no-slip condition is considered, while at the fluid-fluid interface $y=d_{i}$, the velocity and shear stress are considered continuous. Semi-analytical solutions of the problem with initial, boundary and interface conditions have been determined by employing the Laplace transform coupled with the Talbot algorithms for the numerical inverse Laplace transforms. Using the Laplace transform and the finite sine-Fourier transform, the analytical solutions of the same problem have been determined. It has been found that the memory effects are significant in the fluid motion. Furthermore, the fluid with the lowest viscosity has the highest velocity, as expected. 


\section{Compliance with ethical standards}

Conflict of interest On behalf of all authors, the corresponding author states that there is no conflict of interest.

\section{Appendix}

Applying the Laplace transform to Eq. (11), we can write

$\bar{\tau}_{i}(y, \xi)=b_{i} \frac{\partial \bar{u}_{i}(y, \xi)}{\partial y}+\alpha_{i} \xi^{\gamma_{i}} \frac{\partial \bar{u}_{i}(y, \xi)}{\partial y}$

we can rewrite Eq. (61) in equivalent form as

$\frac{\partial \bar{u}_{i}}{\partial y}=\left(\frac{1}{b_{i}+\alpha_{i} \xi^{\gamma_{i}}}\right) \bar{\tau}_{i}(y, \xi)$

Applying the inverse Laplace transform to Eq. (62) gives the velocity gradient in the form of convolution as

$\frac{\partial u_{i}(y, t)}{\partial y}=\psi_{i}\left(t, \gamma_{i}\right) * \tau_{i}(y, t)$

$=\int_{0}^{t} \psi_{i}\left(t-\delta, \gamma_{i}\right) \tau_{i}(y, \delta) d s: i \in I_{n}^{1}$

with $\psi_{i}\left(t, \gamma_{i}\right)=\frac{1}{\alpha_{i}} t^{\gamma_{i}-1} E_{\gamma_{i}, \gamma_{i}}\left(-\frac{b_{i}}{\alpha_{i}} t^{\gamma_{i}}\right)$.

\section{References}

1. Bear J (2013) Dynamics of fluids in porous media. Courier Corporation, Chelmsford

2. Dullien FA (2012) Porous media: fluid transport and pore structure. Academic Press, Cambridge

3. Lake LW (1989) Enhanced oil recovery. Prentice Hall, Englewood Cliffs

4. Satpathi DK, Kumar BR, Chandra P (2003) Unsteady-state laminar flow of viscoelastic gel and air in a channel: application to mucus transport in a cough machine simulating trachea. Math Comput Model 38(1-2):63-75

5. Gin C, Daripa P (2015) Stability results for multi-layer radial HeleShaw and porous media flows. Phys Fluids 27(1):012101

6. Ward K, Zoueshtiagh F, Narayanan R (2019) Faraday instability in double-interface fluid layers. Phys Rev Fluids 4(4):043903

7. Papaefthymiou ES, Papageorgiou DT (2017) Nonlinear stability in three-layer channel flows. J Fluid Mech 250:433-480

8. Yih CS (1967) Instability due to viscosity stratification. J Fluid Mech 27(2):337-352

9. Le Meur H (1997) Non-uniqueness and linear stability of the one-dimensional flow of multiple viscoelastic fluids. ESAIM: Math Model Numer Anal 31(2):185-211

10. Kalogirou A, Blyth MG (2019) The role of soluble surfactants in the linear stability of two-layer flow in a channel. J Fluid Mech 873:18-48

11. Kim Y, Choi H, Park YG, Jang J, Ha MY (2019) Numerical study on the immiscible two-phase flow in a nano-channel using a molecular-continuum hybrid method. J Mech Sci Technol 33:4291-4302

12. Abd Elmaboud $Y$ (2018) Two layers of immiscible fluids in a vertical semi-corrugated channel with heat transfer: impact of nanoparticles. Results Phys 9:1643-1655

13. Abd Elmaboud Y, Abdelsalam SI, Mekheimer KS, Vafai K (2019) Electromagnetic flow for two-layer immiscible fluids. Eng Sci Technol Int J 22(1):237-248

14. Abdelsalam SI, Bhatti MM (2018) The study of non-Newtonian nanofluid with hall and ion slip effects on peristaltically induced motion in a non-uniform channel. RSC Adv 8(15):7904-7915

15. Sohail M, Naz R, Abdelsalam SI (2020) Application of non-Fourier double diffusions theories to the boundary-layer flow of a yield stress exhibiting fluid model. Physica A 537:122753

16. Khan Z, Islam S, Shah RA, Khan I (2016) Flow and heat transfer of two immiscible fluids in double-layer optical fiber coating. J Coat Technol Res 13(6):1055-1063

17. Hisham MD, Rauf A, Vieru D, Awan AU (2018) Analytical and semi-analytical solutions to flows of two immiscible Maxwell fluids between moving plates. Chin J Phys 56(6):3020-3032

18. Rauf A, Mahsud Y, Mirza IA, Rubbab Q (2019) Multi-layer flows of immiscible fractional Maxwell fluids with generalized thermal flux. Chin J Phys. https://doi.org/10.1016/j.cjph.2019.10.006

19. Rauf A, Mahsud Y, Siddique I (2019) Multi-layer flows of immiscible fractional Maxwell fluids in a cylindrical domain. Chin J Phys. https://doi.org/10.1016/j.cjph.2019.09.015

20. Sadaf H, Abdelsalam SI (2020) Adverse effects of a hybrid nanofluid in a wavy non-uniform annulus with convective boundary conditions. RSC Adv 10(26):15035-15043

21. Zhao D, Hedayat M, Barzinjy AA, Dara RN, Shafee A, Tlili I (2019) Numerical investigation of Fe\$_3\$O\$_4 nanoparticles transportation due to electric field in a porous cavity with lid walls. J Mol Liq 293:111537

22. Joseph DD, Renardy YY (1995) Fundamentals of two-fluid dynamics. J Fluid Mech 282:405

23. Ashraf S, Phirani J (2019) Capillary displacement of viscous liquids in a multi-layered porous medium. Soft Matter 15(9):2057-2070

24. Barannyk LL, Papageorgiou DT, Petropoulos PG, Vanden-Broeck JM (2015) Nonlinear dynamics and wall touch-up in unstably stratified multilayer flows in horizontal channels under the action of electric fields. SIAM J Appl Math 75(1):92-113

25. Funahashi $\mathrm{H}$, Kirkland KV, Hayashi K, Hosokawa S, Tomiyama A (2018) Interfacial and wall friction factors of swirling annular flow in a vertical pipe. Nuclear Eng Design 330:97-105

26. Aliyu AM, Baba YD, Lao L, Yeung H, Kim KC (2017) Interfacial friction in upward annular gas-liquid two-phase flow in pipes. Exp Therm Fluid Sci 84:90-109

27. Caputo M, Fabrizio M (2015) A new definition of fractional derivative without singular kernel. Prog Fract Differ Appl 1(2):1-13

28. Podlubny I (1999) Fractional differential equations mathematics in science and engineering, vol 198. Academic Press, San Diego

29. Caputo $M(1967)$ Linear models of dissipation whose $Q$ is almost frequency independent-II. Geophys J Int 13(5):529-539

30. Xiao-Jun XJ, Srivastava HM, Machado JT (2016) A new fractional derivative without singular kernel. Therm Sci 20(2):753-756

31. Hristov J (2017) Transient space-fractional diffusion with a power-law superdiffusivity: approximate integral-balance approach. Fundam Inf 151(1-4):371-388

32. Ahmed N, Shah NA, Vieru D (2019) Two-dimensional advection-diffusion process with memory and concentrated source. Symmetry 11(7):879

33. Abate J, Valko PP (2004) Multi-precision Laplace transform inversion. Int J Numer Methods Eng 60:979-993. https://doi. org/10.1002/nme.995 
34. Lorenzo CF, Hartley TT (1999) Generalized functions for the fractional calculus. NASA/TP-1999-209424/REV1

35. Atangana A (2017) Fractional operators with constant and variable order with application to geo-hydrology. Academic Press, Cambridge

36. Arshad M, Choi J, Mubeen S, Nisar KS, Rahman G (2018) A new extension of the Mittag-Lefler function. Commun Korean Math Soc 33(2):549-560. https://doi.org/10.4134/CKMSC170216

37. Brian D (2002) Integral transforms and their applications, 3rd edn. Springer, New York
38. Dingfelder B, Weideman JAC (2015) An improved Talbot method for numerical Laplace transform inversion. Numer Algorithms 68:167-183. https://doi.org/10.1007/s11075-014-9895-z

39. Luo L, Shah NA, Alarifi IM, Vieru D (2020) Two-layer flows of generalized immiscible second grade fluids in a rectangular channel. Math Methods Appl Sci 43(3):1337-1348

Publisher's Note Springer Nature remains neutral with regard to jurisdictional claims in published maps and institutional affiliations. 